\title{
Functionalization of Surface-Grafted Polymethylhydrosiloxane Thin Films with Alkyl Side Chains
}

\author{
Thierry Thami $^{1}$, Gihane Nasr ${ }^{1}$, Hanan Bestal $^{1}$, Arie van der Lee ${ }^{1}$ and Bruno Bresson ${ }^{2}$ \\ ${ }^{1}$ Institut Européen des Membranes, ENSCM, Université Montpellier 2, CNRS, CC047, 2 Place \\ Eugène Bataillon, 34095 Montpellier Cedex 5 \\ ${ }^{2}$ LPQ/ESPCI, CNRS, 10 rue Vauquelin, 75231 Paris cedex 05
}

E-mail address: thierry.thami@iemm.univ-montp2.fr, gihane.nasr@iemm.univ-montp2.fr, vdlee@iemm.univ-montp2.fr, bruno.bresson@espci.fr

Corresponding author: Thierry Thami, Institut Européen des Membranes, Université Montpellier 2, CNRS, ENSCM, CC047, 2 Place Eugène Bataillon, 34095 Montpellier cedex 5, France (thierry.thami@iemm.univ-montp2.fr) 


\section{ABSTRACT}

Thin films of crosslinked polymethylhydrosiloxane (PMHS) have been grafted on silica using the sol-gel process allowing further functionalization by effective quantitative hydrosilylation of $\mathrm{SiH}$ groups by olefins within the network. Postfunctionalization gives the polysiloxane network with $n$ alkyl side chains. The PMHS coating was prepared by room temperature polycondensation of a mixture of methyldiethoxysilane $\mathrm{HSiMe}(\mathrm{OEt})_{2}$ monomer and triethoxysilane $\mathrm{HSi}(\mathrm{OEt})_{3}(\mathrm{TH})$ as crosslinker. The surface-attached films are chemically stable and covalently bonded to the silica surface. Subsequently, films were functionalized without delamination. We showed by FTIR spectroscopy how the crosslinking ratio and the molecular size of the alkenes precursors influence the extent of the hydrosilylation reaction of $\mathrm{SiH}$ groups in the PMHS network. We have determined that quasi-full olefin addition catalyzed by a platinum complex occurred within soft networks of less than $5 \%$ TH with 1 -alkenes $\mathrm{CH}_{2}=\mathrm{CH}\left(\mathrm{CH}_{2}\right)_{\mathrm{n}-2} \mathrm{CH}_{3}$ of various alkyl chain lengths $(n=5,11,17)$. Powders of PMHS gel were also modified with 1-alkenes by hydrosilylation. The SiH groups within the soft gel ( $5 \%$ crosslinked) were fully functionalized as shown by ${ }^{29} \mathrm{Si}$ and ${ }^{1} \mathrm{H}$ solid state NMR. The structure of functionalized polysiloxane with $n$-octadecyl and $n$-dodecyl side chains was studied by FTIR, wide angle X-ray diffraction and DSC showing crystallization of the long $n$-alkyl chains in the network.

Key words: Thin films; crosslinking; silicones; hydrosilylation; surface functionalization 


\section{INTRODUCTION}

Surface functionalization of a solid substrate is an important way to obtain materials with improved interfacial performance. A number of synthetic routes have been developed for surface modification of materials used for separation, sensors, wetting and adhesion, smart surfaces with sensing and responsive properties, biomedical devices and other applications. ${ }^{1-4}$ Hence, it is a challenge to achieve crosslinked materials that are grafted to the substrate and that allow further chemical surface modification with molecules or various functionalities.

One important chemical pathway for preparing polymer thin films is the sol-gel process of silicon alkoxide monomers $R_{n} \operatorname{Si}\left(\mathrm{OR}^{\prime}\right)_{4-n}(n=1$ or $2 ; R$ is currently an organic group). We focused mainly on SiH substituted silicon alkoxide precursors $(R=\mathrm{H})$ because they have high reactivity toward hydrolytic polycondensation in sol-gel process $^{5-8}$ and usually give material with a high extent of condensation in contrast to precursors substituted with bulky organic groups. ${ }^{9}$ These $\mathrm{SiH}$ alkoxide monomers lead consequently to lower residual silanols in the final gel. In this context, we developed previously a sol-gel route to tailor surface grafted elastomeric polymethylhydrosiloxane-like (PMHS) networks ${ }^{10}$ before focusing in a second step on the postfunctionalization of these surface grafted thin films by hydrosilylation.

Sol-gel PMHS materials was previously crosslinked by room temperature polycondensation of a mixture of methyldiethoxysilane $\mathrm{HSiMe}(\mathrm{OEt})_{2}(\mathrm{DH})$ silicon alkoxide monomers and triethoxysilane $\mathrm{HSi}(\mathrm{OEt})_{3}(\mathrm{TH})$ as crosslinker. The method allowed us to synthesize a soft elastomeric PMHS network as powdered gels or as films deposited on surfaces in a wide range of DH/TH composition, from $50 / 50$ to $99 / 1$, with continuous variations of their mechanical properties. ${ }^{10}$ The copolymerization of DH/TH mixtures resulted in the formation of a fully condensed, homogeneous and highly hydrophobic 3D network as a copolymer formed by polymethylhydrosiloxane (PMHS) segments interconnected by randomly dispersed $\mathrm{SiHO}_{3 / 2}$ crosslink subunits. No uncondensed $\mathrm{SiOH}$ or SiOEt species were found in such a networked siloxane polymer.

On the other hand, a variety of functionalized elastomeric silicones have been developed for example for protective coating, ${ }^{11-13}$ gas separation or biomedical application ${ }^{14}$ and were crosslinked by a wide range of curing methods. ${ }^{13}$ Thus, the facile formation of the elastomeric sol-gel networked 
PMHS under ambient condition and its subsequent postfunctionalization by hydrosilylation is a new versatile synthetic route of interest in these areas to obtain elastomeric network polymers.

In addition, crosslinked PMHS films achieved by the sol-gel method are strongly attached to oxide surfaces (e.g. glass, quartz) through SiOSi bonds with surface reactive silanol sites. ${ }^{10}$ The covalent bonding to surfaces is an advantage for applications to avoid delamination of films and to assure a long-term chemical stability. In the case of $\mathrm{Si}-\mathrm{H}$ precursors, $\mathrm{SiH}$ groups could also react with silanol groups of the substrate involving the surface-catalyzed cleavage of $\mathrm{Si}-\mathrm{H}$ groups with surface water ${ }^{15}$ followed by $\mathrm{Si}-\mathrm{O}-\mathrm{Si}$ linkages which leads to an increase in the crosslinking density of the film near the silica surface. Similar reactions of surface attachment resulting from hydrolysis of a part of the $\mathrm{Si}-\mathrm{H}$ group on hydrated silica have been reported for various $\mathrm{Si}-\mathrm{H}$ containing polymers ${ }^{16-18}$ and for organosilicon hydrides $\mathrm{RSiH}_{3}{ }^{19}$

Hydrosilylation is an important industrial reaction in polysiloxane synthesis. It is well known that addition of a terminal double bond to $\mathrm{Si}-\mathrm{H}$ groups can be usually carried out almost quantitatively in bulk or in solution, catalyzed by a platinum complex. ${ }^{20-22}$ Moreover, the reaction is irreversible and the $\mathrm{Si}-\mathrm{C}$ bonds formed are hydrolytically stable. However, the reaction involves steric hindrance and, therefore, can lead frequently to incomplete conversion of the $\mathrm{Si}-\mathrm{H}$ groups.

Methods to modify the surface of silica powders or particles were developed by Peseck ${ }^{23-26}$ and by Brook $^{27-29}$ with triethoxysilane coupling agent $\mathrm{TH}$ to postfunctionalize the resulting $\mathrm{T}^{\mathrm{H}}$ layer by subsequent hydrosilylation of $\mathrm{Si}-\mathrm{H}$ groups for application in chromatography ${ }^{26}$ and in catalysis. ${ }^{28,29}$ In fact, the $\mathrm{TH}$ thin layer formed by silanization on the silica surface was dense because of the trifunctionality of the alkoxide precursor which leads to a networked polymer with a high degree of crosslinking. On other hand, silica gel was modified by Fukui by thermal catalytic polymerization of methylcyclosiloxane precursor to achieve an $\mathrm{SiH}$ containing polymeric thin layer ${ }^{18}$ further modified by hydrosilylation. ${ }^{30-32}$ However, the detailed structure of the thin polymeric film (less than $1 \mathrm{~nm}$ ) obtained by this method remains unclear due to the difficulty of analysis. Thereby, surface modification of silica gel and particles were reported to modify the interfacial properties by deposition of an ultra thin polymeric layer. 
The sol-gel synthesis of an elastomeric networked PHMS that we proposed ${ }^{10}$ allowed the difficulties mentioned above to be overcome. Firstly, the number and accessibility of functional $\mathrm{SiH}$ groups were controlled for subsequent grafting of the desired function or molecules. Secondly, the sol-gel reactions can be extended on flat surfaces to obtain films of controlled thickness by spin- or dip-coating for applications such as membranes, sensors or drug delivery. The addition of a terminal double bond to the silicon hydride species $\mathrm{P}-\mathrm{SiH}\left(\mathrm{P}=\mathrm{T}^{\mathrm{H}}\right.$-crosslinked PMHS) involves reaction between alkenes and $\mathrm{Si}-\mathrm{H}$ groups derivatized from both $\mathrm{DH}$ and $\mathrm{TH}$ subunits:

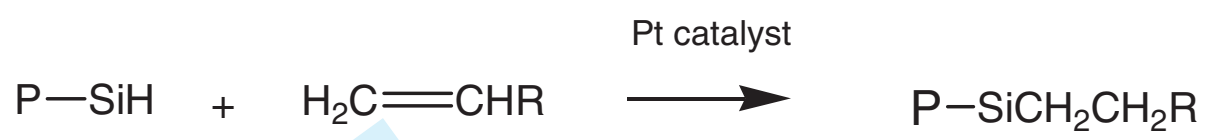

Our aim was to show how the crosslinking density affects the swelling, the stability of the surfacegrafted film and the diffusion of reagent and platinum catalyst in order to obtain the full conversion of SiH groups into networked PHMS by the hydrosilylation reaction.

In this work, the virgin PMHS thin films were studied as a function of the crosslinking density using FTIR spectroscopy. The network reactivity toward hydrosilylation was then studied as a function of both crosslink density and length of the alkenes $\mathrm{CH}_{2}=\mathrm{CH}\left(\mathrm{CH}_{2}\right)_{n-2} \mathrm{CH}_{3}$ (from $n=5$ to 17). The reaction (1) should result in the formation of the main anti-Markovnikov adduct for long alkyl chains. For sake of comparison, the addition was achieved with networked PMHS as thin films on silicon wafer support or as particles after grinding the gel structure. The $n$-alkyl functionalization and the effect of the crosslinking density on the hydrosilylation reaction for PMHS95 and PMHS70 network (thin film and powder) is discussed (PMHS95 and PMHS70 denote 5\% and 30\% THcrosslinked polymers, respectively). The characterization of the $n$-alkyl functionalized PMHS95 polymer as powder was performed by solid state Nuclear Magnetic Resonance $\left({ }^{1} \mathrm{H}\right.$ and ${ }^{29} \mathrm{Si}$ MAS NMR), FTIR, X-ray diffraction and DSC analysis. In this way, the ability of the side chains to crystallize was studied.

\section{EXPERIMENTAL}

\section{Chemicals}

Both the precursors methyldiethoxysilane $\mathrm{HSi}\left(\mathrm{CH}_{3}\right)\left(\mathrm{OCH}_{2} \mathrm{CH}_{3}\right)_{2}$ and triethoxysilane $\mathrm{HSi}\left(\mathrm{OCH}_{2} \mathrm{CH}_{3}\right)_{3}$ were purchased from $\mathrm{ABCR}$ and used as received. Water used for cleaning the 
substrates was obtained with a Milli-Q water purification apparatus (Millipore). Absolute ethanol was dried over $4 \AA$ molecular sieve. The catalyst trifluoromethanesulfonic acid $\mathrm{CF}_{3} \mathrm{SO}_{3} \mathrm{H}$ and 1dodecene (purity 95\%) were purchased from Aldrich; 1-hexene (purity 97\%) from Acros Organics and 1-octadecene (purity 95\%) from Fluka. All solvents, $\mathrm{CHCl}_{3}, \mathrm{CCl}_{4}$, toluene or $n$-hexane, were of synthesis grade purity. When required, toluene and other solvents were dried with calcium hydride and distilled before use. Hexachloroplatinic (IV) acid hexahydrate $\mathrm{H}_{2}\left[\mathrm{PtCl}_{6}\right]\left(\mathrm{H}_{2} \mathrm{O}\right)_{6}$ and the platinum-divinyltetramethyldisiloxane complex in xylene (PC072) (platinum concentration of about $0.1 \mathrm{M}$ assuming $2.4 \mathrm{wt} \% \mathrm{Pt}$ in xylene), also known as the Karstedt catalyst, were purchased from ABCR. The Speier catalyst was prepared by dissolving hexachloroplatinic acid (0.1 g) in 2-propanol $(1 \mathrm{~mL})$ and was stored in a freezer.

\section{Synthesis of Thin Films}

\section{Substrate Cleaning and Activation}

Silicon wafers $\mathrm{Si}(100)$ from MEMC Electronic materials were used as substrates and were typically cut into rectangular strips of about $2 \times 2.5 \mathrm{~cm}^{2}$ for dip-coating, or square strips of $2 \times 2 \mathrm{~cm}^{2}$ for spin-coating deposition. To properly anchor the final PMHS thin film by covalent $\mathrm{Si}-\mathrm{O}-\mathrm{Si}$ linkage on native oxide silica (thickness $\sim 1.8 \mathrm{~nm}$ ), the silicon wafers were first cleaned and activated using the previously described procedure. ${ }^{10}$

\section{Synthesis of Crosslinked PMHS}

The crosslinking density of PMHS thin films was tuned by room temperature sol-gel polymerization of $\mathrm{DH} / \mathrm{TH}$ liquid mixtures deposited by dip or spin coating onto freshly activated silicon wafer. The dip-coating procedure was previously detailed for various DH/TH liquid mixtures from 50/50 to $95 / 5(\mathrm{~mol} \%) .{ }^{10}$ Alternatively, typical parameters for spin-coating deposition are presented here for the preparation of homogeneous thin films samples of the elastomeric PMHS network starting from $\mathrm{DH} / \mathrm{TH}$ in the range $85 / 5-99 / 1$ (mol \%). A typical sol preparation was as follows: from a mixture $\alpha / 1-\alpha$ of $\mathrm{DH} / \mathrm{TH}$, the monomers were polymerized at about $4 \mathrm{M}$ concentration in EtOH (molar ratio $[\mathrm{EtOH}] /[\mathrm{Si}]=1$ ), with 0.5 equivalent of water (hydrolysis ratio $h$ $\left.=\left[\mathrm{H}_{2} \mathrm{O}\right] /[\mathrm{SiOEt}]=0.5\right)$ according to the sol-gel preparation Scheme: 
$\alpha \mathrm{HSi}\left(\mathrm{CH}_{3}\right)(\mathrm{OEt})_{2}+(1-\alpha) \mathrm{HSi}(\mathrm{OEt})_{3}+(3-\alpha) / 2 \mathrm{H}_{2} \mathrm{O}$

$$
\rightarrow\left[\mathrm{SiH}\left(\mathrm{CH}_{3}\right) \mathrm{O}_{2 / 2}\right]_{\alpha}\left[\mathrm{SiHO}_{3 / 2}\right]_{1-\alpha}+(3-\alpha) \mathrm{EtOH}
$$

Trifluoromethanesulfonic acid $\mathrm{CF}_{3} \mathrm{SO}_{3} \mathrm{H}(1 \mathrm{M}$ in absolute ethanol) was used as catalyst $(0.5$ $\mathrm{mmol} / \mathrm{mol}$ of monomers). The content of catalyst was not higher than $0.05 \%$ to control the kinetics of gelation of the liquid mixture. Indeed, higher content produced inhomogeneous spin-coated layers because gelation was too fast. The resulting clear sols were allowed to age for less than 1 hour with magnetic stirring before spin-coating deposition. The freshly cleaned silicon wafer was purged (2 $\min$ ) in the spin-coater (Laurell Technologies) under a stream of nitrogen $(2 \mathrm{~L} / \mathrm{min}$ ) to avoid air moisture. For all samples, the speed of rotation was $4000 \mathrm{rpm}$ (spin acceleration $2000 \mathrm{rpm} . \mathrm{s}^{-1}$ ) and the time of rotation 30s. This procedure gives layers of reproducible homogeneity and thickness in the submicrometer range as measured by both ellipsometry and infrared spectroscopy. The samples were finally cured at $100{ }^{\circ} \mathrm{C}$ in an oven for 1 h or more. Samples synthesized from 50/50, 70/30 and 95/5 DH/TH sols were respectively denoted PMHS50, PMHS70 and PMHS95.

\section{Hydrosilylation with Olefins}

Prior to hydrosilylation, the thin films were swollen in $\mathrm{CHCl}_{3}$ at room temperature for 30 min to remove any oligomeric PMHS species trapped in the layer. The solvent was removed under a stream of nitrogen. The sample was then dried in an oven at $100{ }^{\circ} \mathrm{C}$ in air for 1 hour. This washing and curing process gives the final densification and thickness of the organic layers as shown by FTIR and ellipsometry measurements. ${ }^{10}$

In a typical preparation of a dodecyl-functionalized film on a silicon wafer, a PMHS95 coated sample (ca. $1 \mu \mathrm{m}$ thickness or less, surface $4 \mathrm{~cm}^{2}$ ) was placed in a solution of 1 -dodecene $(1 \mathrm{ml}$, density $0.758 \mathrm{~g} / \mathrm{mL}, 4.5 \mathrm{mmol})$ and the Karstedt catalyst $\left(1 \mu \mathrm{L}, 10^{-4} \mathrm{mmol}\right)$ in dry toluene or $n$ hexane $(10 \mathrm{~mL})$ for $2-3$ hours at $60-70{ }^{\circ} \mathrm{C}$. After the sample was removed from the reaction mixture, it was rinsed with toluene (twice) and chloroform (twice) to remove any physisorbed material. The sample was then dried in a stream of nitrogen and cured in the oven at $100{ }^{\circ} \mathrm{C}$ in air for 1 hour. The reaction was normally complete or near completion within $2-3$ hours. This was determined by complete disappearance of the $\mathrm{Si}-\mathrm{H} \mathrm{IR}$ absorption at about $2169 \mathrm{~cm}^{-1}$. If required, the 
procedure was repeated until complete, or almost complete, absence of unreacted $\mathrm{Si}-\mathrm{H}$ groups as detected by FTIR spectroscopy.

We also obtained a quantitative result by using $0.1 \mathrm{~mL}$ of dodecene instead of $1 \mathrm{~mL}$. In both cases, the olefin was in large excess with respect to available $\mathrm{SiH}$ on a grafted PMHS thin film of thickness $1 \mu \mathrm{m}$ or less.

Alternatively, docecyl-grafted PMHS95 films were prepared using the Speier catalyst $(1 \mu \mathrm{L})$ and 1dodecene $(0.1 \mathrm{~mL})$ yielding the same results as with FTIR spectroscopy. Toluene or $n$-hexane were preferred as the swelling solvent rather than $\mathrm{CHCl}_{3}$ or $\mathrm{CCl}_{4}$ for hydrosilylation of $5 \%$ crosslinked PMHS film because no delamination of the $1 \mu \mathrm{m}$-thick surface-attached PMHS95 layer occurred upon reaction with olefin in these solvents.

For the preparation of hexyl- and octadecyl-functionalized films, a similar procedure was followed with 1-hexene (density $0.678 \mathrm{~g} / \mathrm{mL})$ and 1 -octadecene $(0.789 \mathrm{~g} / \mathrm{mL})$, respectively.

For the highly crosslinked network PMHS70 ( $c a .1 \mu \mathrm{m}$ thickness or less), the hydrosilylation reaction was incomplete whatever the alkene used, 1-hexene, 1-dodecene or 1-octadecene, as determined by examination of the $\mathrm{Si}-\mathrm{H}$ band absorption by FTIR (see Results and Discussion section). In the latter case, attempts to use other good swelling solvents such as $\mathrm{CCl}_{4}$ instead of toluene, did not improve the reaction with 1-alkenes even when the reaction was repeated once more on the same sample as shown by infrared spectroscopy.

\section{Analysis of Film Properties}

The thickness of the PMHS thin film deposited by dip- or spin-coating on the silicon surface and that of alkyl functionalized PMHS were determined by an ellipsometer (Plasmos SD 2300) at $70^{\circ}$ incidence angle. Refractive indices used to calculate the thickness were $1.40^{10}$ for PMHS, $1.45^{33}$ for alkyl functionalized PMHS95, $1.457^{34}$ for $\mathrm{SiO}_{2}$ and $3.871-0.016 i^{34}$ for $\mathrm{Si}$. For polymer films of thickness ca. $1 \mu \mathrm{m}$, measurements were done at $70.0^{\circ}$ and $50.0^{\circ}$ incidence angles to calculate the thickness value. 
Transmission IR absorption spectra were recorded on thin films using a Nicolet Nexus FTIR spectrometer in the $4000-400 \mathrm{~cm}^{-1}$ range with 64 scans and $4 \mathrm{~cm}^{-1}$ resolution. A background spectrum was recorded using an unmodified silicon wafer in air.

The film thicknesses $(e)$ of dip- or spin-coated PMHS thin films on silicon wafer were also measured as previously described ${ }^{10}$ using transmission IR by assuming the IR spectrum obeys the Beer Lambert law. The thickness of PMHS95 films were calculated from the absorbance of Si-O and $\mathrm{Si}-\mathrm{H}$ peaks at 1100 and $2169 \mathrm{~cm}^{-1}$, respectively, $A_{1100}=\alpha_{S i O} e$ and $A_{2169}=\alpha_{S i H} e$ where $\alpha_{S i O}$ $\left(0.37 \mu^{-1}\right)$ and $\alpha_{S i H}\left(0.27 \mu \mathrm{m}^{-1}\right)$ being the absorption coefficients calibrated by ellipsometry. To confirm the calibration in the micrometer range, the thickness was measured from the cross-section using scanning electron microscopy (Hitachi). The values $\alpha_{S i O}$ used to calculate the thickness of crosslinked PMHS were $0.47 \mu \mathrm{m}^{-1}$ for PMHS50, $0.41 \mu \mathrm{m}^{-1}$ for PMHS70 and $0.37 \mu \mathrm{m}^{-1}$ for low crosslinked PMHS films in the range of DH/TH composition from 85/15 to 99/1 (mol \%).

\section{Synthesis of Powders}

\section{Synthesis of Crosslinked PMHS}

The preparation of the PMHS95 gel was as follows: in a 95/5 (mol \%) mixture of the monomers $\mathrm{DH} / \mathrm{TH}$, the solvent EtOH $([\mathrm{EtOH}] /[\mathrm{Si}]=1)$, the catalyst $\mathrm{CF}_{3} \mathrm{SO}_{3} \mathrm{H}(3 \mathrm{mmol} / \mathrm{mol}$ of monomers $)$ and then water $(h=0.5)$ were added under vigorous stirring. The resulting clear sols were aged with magnetic stirring for 24 hours and then gels were obtained after about 5-6 days of drying in an open vessel at room temperature. The bulk gel was then ground into a fine powder with an agate mill.

The PMHS70 gels were obtained after 3 hours under similar conditions by sol-gel polymerization of a DH/TH 70/30 mixture using $0.5 \mathrm{mmol}$ of catalyst per mol of monomers.

\section{Hydrosilylation with Olefin}

For hydrosilylation of 1-hexene, 1-dodecene and 1-octadecene, the polymeric PMHS95 and PMHS70 gels were used in the form of a finely ground powder. The powder particles have a size between 10 to $150 \mu \mathrm{m}$ as observed by scanning electron microscopy. Prior to hydrosilylation, the powdered gels were first cured at $100{ }^{\circ} \mathrm{C}$ in air in the oven for one hour or more and then purified by 
Soxhlet extraction with $\mathrm{CCl}_{4}$ to remove PMHS oligomers trapped in the network. The washed powders were finally dried at $100{ }^{\circ} \mathrm{C}$ in the oven in air.

In a typical preparation of a dodecyl-functionalized PMHS95 powder, to a mixture of 1-dodecene (3 $\mathrm{mL}, 13.5 \mathrm{mmol})$ and PMHS95 powders $(0.4 \mathrm{~g}, 6.75 \mathrm{mmol})$ swollen in toluene $(20 \mathrm{~mL})$, the Karstedt catalyst $\left(15 \mu \mathrm{L}, 1.510^{-3} \mathrm{mmol}\right)$ was added with magnetic stirring. The reaction was allowed to proceed under reflux overnight. The reaction was judged to be complete by monitoring the disappearance of the $\mathrm{Si}-\mathrm{H}$ absorption in the IR spectrum at $2169 \mathrm{~cm}^{-1}$. The final solid was filtered and washed thrice with toluene. After the solvent was removed, the solid was dried at $50{ }^{\circ} \mathrm{C}$ overnight.

A similar procedure was used for the preparation of an octadecyl-functionalized PMHS95 powder. To a mixture of 1-octadecene $(4.3 \mathrm{~mL}, 13.4 \mathrm{mmole})$ and PMHS95 powders $(0.4 \mathrm{~g}, 6.75 \mathrm{mmol})$ swollen in $\mathrm{CCl}_{4}(20 \mathrm{~mL})$, the Karstedt catalyst $\left(15 \mu \mathrm{L}, 1.510^{-3} \mathrm{mmol}\right)$ was added with magnetic stirring. The reaction was allowed to proceed under reflux overnight. To the resulting oily liquid-gel was added $\mathrm{MeOH}$ ( $\mathrm{ca}$. same volume as $\mathrm{CCl}_{4}$ ) and a white solid gel settled to the bottom of the tube. The supernatant phase was removed. The solvent $\mathrm{CCl}_{4}-\mathrm{MeOH}$ procedure was performed twice. After the final addition, the solid gel was filtered, washed with methanol and dried at $50{ }^{\circ} \mathrm{C}$ overnight.

Hydrosilylation of the more rigidly functionalized PMHS70 powder under similar conditions with 1-hexene or 1-octadecene gives incomplete reaction as determined by FTIR spectroscopy (see Result and Discussion section).

\section{Analysis of powders}

High-resolution solid state NMR spectra $\left({ }^{1} \mathrm{H}\right.$ and $\left.{ }^{29} \mathrm{Si}\right)$ were recorded with an ASX 500 Bruker spectrometer. The experimental conditions that we used for the different techniques are as follows. ${ }^{1} \mathrm{H}$ magic angle spinning (MAS): single pulse experiment, $4 \mu \mathrm{s}$ pulse length, recycling delay $10 \mathrm{~s}$, spinning frequency of $10 \mathrm{kHz}$, and 32 scans accumulation. ${ }^{29} \mathrm{Si}$ MAS: single pulse experiment with proton decoupling, $4 \mu$ s pulse, recycle delay of $30 \mathrm{~s}$, spinning frequency $5 \mathrm{kHz}$, with 500 scans. IR spectra were recorded on powdered samples with the dried $\mathrm{KBr}$ pellet technique by using a Nicolet Nexus FTIR spectrometer in the $4000-400 \mathrm{~cm}^{-1}$ range with 64 scans and $4 \mathrm{~cm}^{-1}$ resolution the 
background spectrum being recorded in air. Wide angle diffractograms were obtained on a PanAnalytical X'pert Pro diffractometer using Ni-filtered $\mathrm{Cu} \mathrm{K} \alpha$ radiation. Differential scanning calorimetry (DSC) analyses were performed by using a DSC 2920 (TA Instruments) calorimeter calibrated with an indium standard. Samples of $c a$. 3-5 $\mathrm{mg}$ encapsulated in aluminium pans were run under nitrogen at a heating rate of $10{ }^{\circ} \mathrm{C} / \mathrm{min}$ from -140 to $150{ }^{\circ} \mathrm{C}$.

\section{RESULTS AND DISCUSSION}

\section{Synthesis and Characterization of Crosslinked PMHS Thin Films}

\section{Film deposition}

In the first step, the room temperature sol-gel deposition of a polymethylhydrosiloxane (PMHS) network of controlled thickness in the submicrometer range was performed on the silica surface of the silicon wafer, using spin-coating or dip-coating methods. It results in thin films which presented a good optical homogeneity. Table 1 shows their typical thicknesses and most of the characteristic FTIR data from various DH/TH sol compositions.

The sol-gel procedure allows fine adjustment of the crosslinking density of the gel network by changing the TH crosslinker ratio in the preparation scheme (2) of the starting DH/TH sol from 1 to $50 \%$. The crosslinking density determines the extent of swelling, the mechanical properties, the effective pore size of the gel, and the stability of the covalently grafted material on the silica surface. These properties in turn determine the suitability of the crosslinked PMHS polymers for hydrosilylation synthesis. The resulting PMHS network structure can be described as a copolymer formed by crosslinked polymethylhydrosiloxane segments - $\left(\mathrm{SiHCH}_{3} \mathrm{O}_{2 / 2}\right)$ - where the reticulation points are randomly dispersed trifunctional $\mathrm{SiHO}_{3 / 2}\left(\mathrm{~T}^{\mathrm{H}}\right)$ crosslinker subunits. The crosslinking density of the 3D network structure can be defined either as the crosslinker concentration $\mu$ or as the chain concentration of the PMHS segment between crosslinks v. Assuming the PMHS gel is fully $\mathrm{T}^{\mathrm{H}}$-crosslinked and neglecting the formation of cycles, it can be shown from simple geometric consideration that:

$$
\mu=\frac{2}{f} v
$$


where $f=3$ is the functionality of the crosslinkers. Then for a mixture $\alpha /(1-\alpha)$ of $\mathrm{DH} / \mathrm{TH}$, the average number of repeat unit $\mathrm{N}$ between two crosslinkers in PMHS segment is:

$$
N=\frac{2 \alpha}{3(1-\alpha)}
$$

where $\alpha$ is the molar fraction of the DH monomer. The upper limit mesh size $\xi_{\max }$ of the gel corresponds to fully stretched PMHS chains in the all-trans conformation. Then, assuming uniform distribution of mesh size, for the extended PMHS segments $\left(\mathrm{SiHCH}_{3} \mathrm{O}_{2 / 2}\right)_{\mathrm{N}}$ the numerical value of $\xi_{\text {max }}$ is given by:

$$
\xi_{\max }(\AA)=3.12(N+1)
$$

where every $\mathrm{Si}-\mathrm{O}$ subunit contributes $1.56 \AA$ to the mesh size (for an $\mathrm{Si}-\mathrm{O}$ bond length of $1.66 \AA$ and an Si-O-Si angle of $140^{\circ}$ ). In Table 1 , the estimated values of $N$ and $\xi_{\text {max }}$ were then calculated from eq 4 and 5 for the various $\mathrm{DH} / \mathrm{TH}$ compositions. It can be predicted qualitatively that small molecules can diffuse in such networks, especially molecules of good solvents for polymethylsiloxane polymers (e.g. chloroform, toluene, hexane). Thus, elastomeric networks constructed from DH molar compositions greater than $85 \%$ are particularly adapted for the alkene postfunctionalization of the PMHS network.

The FTIR characteristic of soft fully $\mathrm{T}^{\mathrm{H}}$-crosslinked thin films (Table 1) and a previous study of their Young's moduli by atomic force microscopy ${ }^{10}$ both indicate qualitatively that the number of repeat units between crosslinks $(N)$ increases continuously by decreasing the concentration of TH crosslinker to as little as $1 \%($ mol \%). However, additional measurements should be made such as swelling experiments in solvent to provide an experimental value for the molecular weight between crosslinks which should be further related to the alkene functionalization density by hydrosilylation of such crosslinked networks.

\section{Characterization of PMHS by Infrared-spectroscopy}

The structure of the PMHS thin films was investigated by infrared spectroscopy (Figure 1) showing that immediately after their preparation $\mathrm{DH} / \mathrm{TH}$ mixtures react totally to yield fully crosslinked networks as previously shown from an NMR study of the powder. Indeed, we observed strong doublet bands located at $1000-1100 \mathrm{~cm}^{-1}$ corresponding to the asymmetric stretching of $\mathrm{Si}-$ 
O-Si bonds. On increasing the molar fraction of the DH precursor from 50 to $95 \%$, the intensities of the $1100-\mathrm{cm}^{-1}$ bands ( $\mathrm{Si}-\mathrm{O}-\mathrm{Si}$ ), chosen for thickness calibration, are slightly decreased by about $20 \%$ (see Experimental part). This is due to the lower density of $\mathrm{Si}-\mathrm{O}$ vibration groups in the low crosslinked gels as might be expected. Of particular relevance, the flat spectrum in the $v_{\mathrm{O}-\mathrm{H}}$ region $3200-4000 \mathrm{~cm}^{-1}$ confirmed the absence of hydroxy group.

The $\mathrm{C}-\mathrm{H}$ stretching absorptions of $\mathrm{CH}_{3}$ group in $\mathrm{SiCH}_{3}$ of $\mathrm{DH}$ subunits around 2910 and $2965 \mathrm{~cm}^{-}$ ${ }^{1}$ are weak. However, an intense and sharp band is found at $1262\left( \pm 2 \mathrm{~cm}^{-1}\right)$ for the symmetrical $\mathrm{CH}_{3}$ deformation $\delta_{\mathrm{SiCH}_{3}}$ of the $\mathrm{DH}$ subunits which is the single most characteristic band for the organosilicon compound (Table 1). Other bands have also been attributed to the methyl group ${ }^{35}$ in $\mathrm{SiCH}_{3}$ at about $1410\left(\delta_{\mathrm{a}} \mathrm{SiCH}_{3}\right.$ asymmetric deformation) and $770\left(\delta_{\mathrm{r}} \mathrm{SiCH}_{3}\right.$ rocking mode $)$ by comparison with liquid oligomers of PMHS.

The FTIR spectra exhibit as expected intense $\mathrm{Si}-\mathrm{H}$ bands ${ }^{35}$ for stretching vibrations in the 2200 $2300 \mathrm{~cm}^{-1}$ region and for bending vibrations $\delta_{\mathrm{SiH}}\left(891\right.$ and $\left.835 \pm 5 \mathrm{~cm}^{-1}\right)$. In addition, the $v_{\mathrm{SiH}}$ stretching peak of the DH subunit is moved to lower frequency with respect to the $\mathrm{TH}$ crosslinker subunit because of the electron-releasing ability of the Si methyl substituent. For instance, the PMHS70 thin film exhibits two $v_{\mathrm{SiH}}$ peaks at 2175 and $2234 \mathrm{~cm}^{-1}$ for DH and $\mathrm{TH}$, respectively (Table 1). Their relative intensities are modified quantitatively in agreement with the DH/TH composition.

By analysis of the $v_{\mathrm{SiH}}$ stretching vibration bands we have shown quantitatively that the $\mathrm{SiH}$ groups are preserved in thin films with $\mathrm{DH}$ molar fractions ranging from $50 \%$ to $95 \%$ because the number of $\mathrm{SiH}$ groups in the PMHS segment is correlated with the number of $\mathrm{SiCH}_{3}$ groups. Indeed, the normalized peak area of methyl $\delta_{\mathrm{SiCH}_{3}}$ and of $v_{\mathrm{SiH}}$ stretching bands of $\mathrm{SiHCH}_{3} \mathrm{O}_{2 / 2}$ subunits versus the DH molar fraction are straight lines passing through the origin as plotted in Figure S1 in the Supplementary Information.

An accurate determination of the $v_{\mathrm{SiH}}$ stretching peak position of the $\mathrm{DH}$ subunit versus the $\mathrm{DH}$ molar fraction (Figure 2) also gives information about the chemical composition of the thin films showing the formation of an homogeneous network. The $v_{\mathrm{SiH}}$ frequency of the DH subunit is shifted 
as expected to lower frequency by decreasing the crosslinker $\mathrm{TH}$ concentration from 50 to $1 \%$ following a linear relationship (Fig. 2). The frequency shift for the $\mathrm{Si}-\mathrm{H}$ bond is very sensitive to the inductive effects of the substituents at the silicon atom and therefore to arrangements of the neighboring subunits in the $\mathrm{DH} / \mathrm{TH}$ polymer microstructure. By decreasing the molar fraction of the TH crosslinker the average size of PMHS segments increases, thus $v_{\mathrm{SiH}}$ of $\mathrm{DH}$ is normally shifted to lower frequency because of the electron-releasing ability of DH with respect to the $\mathrm{TH}$ neighboring subunit. This result supports the fact that homogeneous networks are formed using sol-gel process for $\mathrm{DH} / \mathrm{TH}$ mixtures because if phase separation had occurred to a large extent between the cluster of the self-condensed $\mathrm{T}^{\mathrm{H}}$-network and the PMHS homopolymer, no frequency shift would have been observed.

Furthermore, the well-designed doublet-like $v_{\mathrm{SiH}}$ band structure in the $2100-2300 \mathrm{~cm}^{-1}$ region corresponding to $\mathrm{Si}-\mathrm{H}$ bonds in the $\mathrm{SiHCH}_{3} \mathrm{O}_{2 / 2}$ and $\mathrm{SiHO}_{3 / 2}$ subunits, was analyzed quantitatively by curve fitting (see Supplementary Information). For instance, a Gauss-Lorentz peak fitting procedure applied to the $v_{\mathrm{SiH}}$ band structure of the PMHS90 thin film showed that four curves are needed to model the $\mathrm{Si}-\mathrm{H}$ stretching vibration (Figure S2 in Supplementary Information). An attribution of the various curves is given in Figure S3 in the Supplementary Information. In this way, the $\mathrm{Si}-\mathrm{H}$ band corresponding to $\mathrm{DH}$ subunits is fitted with two curves. The curve with lowest wavenumber is attributed to self-condensed DH subunits in the PMHS segment in agreement with values found for PMHS homopolymers. ${ }^{35,36}$ The curve with highest wavenumber is attributed to DH co-condensed species with TH subunits. Their integrated intensities are in good agreement with the proposed structure for the PMHS90 composition (Figure S3). Analysis of the $\mathrm{Si}-\mathrm{H}$ band corresponding to the TH subunits can also be fitted with two curves attributed to various cocondensed species with DH subunits. No self-condensed TH species were detected by comparison with gels ${ }^{6}\left(\mathrm{HSiO}_{3 / 2}\right)_{n}$ prepared under similar conditions or with $\mathrm{T}^{\mathrm{H}}$-resin. ${ }^{37}$ Neither were oligomeric species $^{37}$ such as $\mathrm{T}_{8}^{\mathrm{H}}\left(\mathrm{HSiO}_{3 / 2}\right)_{8}$ or $\mathrm{T}_{10}^{\mathrm{H}}\left(\mathrm{HSiO}_{3 / 2}\right)_{10}$ observed. This result clearly rules out any extensive formation of TH clusters in the PMHS90 gel. 


\section{Stability of the Surface-Grafted PMHS Thin Films}

One advantage of the sol-gel process is that crosslinking and surface attachment can be simultaneously performed to bind the final thin film by covalent $\mathrm{Si}-\mathrm{O}-\mathrm{Si}$ linkages on the native silicon oxide $\mathrm{SiO}_{2}$ layer of the silicon wafer substrate. To properly anchor the film to the substrate through covalent chemical bonds, the silica surface was first treated with the 'piranha' solution to increase the surface density of silanol groups before film coating. Under these conditions, the surface chemistry is well defined by the presence of reactive silanol sites on silica $\mathrm{SiO}_{2}$ layer, ${ }^{38}$ about $510^{14}$ silanol groups per $\mathrm{cm}^{2}$, with which molecules in the liquid sol can react. These unreacted pendant silanol groups can bind to the molecules of the surface reactive silanol sites by forming $\mathrm{Si}-\mathrm{O}-\mathrm{Si}$ linkages.

Films of various compositions deposited either by spin- or dip-coating are really surface-attached and chemically stable. For instance, the films are not delaminated upon swelling in good solvents $\left(\mathrm{CHCl}_{3}\right.$, toluene, hexane) and cannot be removed from the surface even by rigorous extraction with boiling solvent.

The covalent bonding of the film is a key point for the robustness of the surface coating. The density of covalent $\mathrm{Si}-\mathrm{O}-\mathrm{Si}$ bonds between the silica surface and the siloxane network is expected to increase with the degree of crosslinking. However, it was found that the attachment to the silica surface is effective even for lightly crosslinked networks such as PMHS99 which are stable in swelling organic solvents. As previously mentioned, the hydrolysis of a part of the $\mathrm{SiH}$ bonds and reaction with silanol groups in the vicinity of the silica substrate probably also play a role in the mechanism of surface attachment.

\section{Synthesis and Characterization of Alkyl Functionalized PMHS}

\section{Effect of Crosslinking Ratio}

The conversion of $\mathrm{Si}-\mathrm{H}$ groups within the PMHS network was studied by infrared and NMR spectroscopy for various compositions of thin films and powdered gels, and for various alkyl chains. The results are summarized in Table 2. To quantify the hydrosilylation yield, the conversion of $\mathrm{SiH}$ was studied on surface grafted PMHS based on absorption measurements at the $\mathrm{Si}-\mathrm{H}$ stretching band frequency $v_{\mathrm{SiH}}$, by measuring the total integrated intensities in the $2100-2300 \mathrm{~cm}^{-1}$ region of 
reacted and unreacted samples (see next section). Alternatively, to analyze quantitatively the addition of 1-alkene to $\mathrm{SiH}$ bond, the absorbance of the most intense and sharp peaks of the methylene group $\mathrm{CH}_{2}$ in the $2850-2955 \mathrm{~cm}^{-1}$ region can be followed with good sensitivity (Figure S4 in Supplementary Material).

The hydrosilylation reaction can proceed within the PMHS95 network in quasi-quantitative yield except for the octadecyl thin film (Table 2). Films of thickness in the submicrometer range are thus almost fully functionalized by diffusion of reagents into the interior of the films. The lightly crosslinked PMHS95 formed stable elastomeric networks having a porous gel structure which allowed diffusion of reagents and catalyst, especially in the presence of good swelling solvents. The PMHS95 thin films do not delaminate from the surface upon swelling in good solvent and upon hydrosilylation with 1-alkene. Moreover, the PMHS95 powder has a sufficiently stable physical form to permit easy purification by filtration whilst we expect more fragile gels for lower crosslinking ratios, not easy to manipulate and purify.

In contrast, the more rigid PMHS70 network of lower mesh size (Table 1) is not fully functionalized with the 1-alkene series under similar conditions even for the thinnest film $(10 \mathrm{~nm})$. This demonstrates that the facility of functionalization decreases with the crosslink density because the diffusion of alkene molecules is limited. Indeed, Table 2 shows clearly that the conversion of $\mathrm{SiH}$ for the PMHS70 network is a decreasing function of the alkyl chain length. It follows that the smallest alkene $(n=5)$ with a length of the same order as that calculated for the PMHS70 mesh size $\left(\xi_{\max }=8 \AA\right)$ gives a higher percentage of $\mathrm{SiH}$ conversion than dodecene $(n=11)$ or octadecene $(n=$ 17). When increasing the calculated mesh size of the network by a factor of $c a .5$ for the PMHS95 polymer $\left(\xi_{\max }=43 \AA\right.$ ), no effect (or less than for PMHS70) of the alkyl chain length is observed on the network reactivity. This confirms that diffusion of reactant into the network probably plays a major role. The presence of some hindered $\mathrm{SiH}$ reaction sites especially the $\mathrm{T}^{\mathrm{H}}$ subunits can also play a role in the network reactivity especially for the PMHS70 polymer. In addition to the above, other factors including solvent, swelling and compatibility of the alkene reactant with the PMHS polymer are also important parameters regarding network reactivity towards hydrosilylation. 
The reaction was carried out under the optimum conditions using a large excess of olefin with respect to available $\mathrm{SiH}$ on the grafted PMHS thin film and only as little as $210^{-5}$ to $10^{-4}$ mol of platinum catalyst $/ \mathrm{mol}$ of olefin. Only two equivalents of olefins/mol of $\mathrm{SiH}$ were used for hydrosilylation of powdered gels. We used the most common platinum catalyst, divinyltetramethyldisiloxane complex, also known as Karstedt's catalyst, but the Speier catalyst yielded in some cases similar results (see Experimental part and Table 2). In general, the reaction was done in dry solvent such as toluene at temperatures ranging from ambient to $60-70{ }^{\circ} \mathrm{C}$ in a few hours to ensure a good yield. A good swelling solvent such as toluene allowed a rapid diffusion of reagents and catalyst into the organic layer.

After the hydrosilylation reaction of the PMHS95 network, the thickness of the dry layer increased by about $100 \%( \pm 20 \%)$ of the initial thickness as measured by ellipsometry for octadecyl or dodecyl layers. By comparison with standard swelling measurements when the polymers are immersed in a good solvent, the introduction of alkyl side chains into the network by functionalization can act as a "solvent" for the polymer backbones, which induces a swollen structure where the polysiloxane segments become more extended in the functionalized network. It is generally observed that surfaceattached networks swell less in solvent that the non-attached bulk networks with the same crosslinking density because of constrained stress induced by the surface. ${ }^{39}$ In the case of PMHS functionalization, we could expect for these reasons that the $\mathrm{SiH}$ conversion of the elastomeric PMHS95 network can be higher for unconstrained 3D gel than in thin films as shown in Table 2 especially for octadecyl.

Another explanation for the lower yields during hydrosilylation of long chain alkenes is that film reaches its maximum swelling before all the $\mathrm{Si}-\mathrm{H}$ sites have reacted. Further experiments should be done to compare the swelling of the films after hydrosilylation with the maximum swelling of the films when immersed in a good solvent.

We propose a structure of the molecular based surface-grafted PMHS network as shown in Figure 3. The networked structure was modelled by PMHS chains between trifunctional crosslink points assuming that all the crosslink points have reacted because unreacted $\mathrm{SiOH}$ or $\mathrm{SiOEt}$ species were not detected by FTIR or NMR before the hydrosilylation reaction. Consequently, we assume that the 
network contains no dangling or pendant chains attached to the network by one end. After hydrosilylation, a swollen structure of the comb-like polysiloxane backbone substituted with alkyl side chains is obtained.

\section{Infrared Spectroscopy}

The spectra of virgin PMHS95 and dodecyl-functionalized PMHS95 thin films are shown in Figure 4. The corresponding dodecyl-functionalized PMHS95 powder showed similar FTIR features. The total conversion of $\mathrm{SiH}$ bonds is clearly shown by the disappearance of both the $v_{\mathrm{SiH}}$ band at $2169 \mathrm{~cm}^{-1}$ and the $\delta_{\mathrm{SiH}}$ bending bands at 891 and $840 \mathrm{~cm}^{-1}$ (Fig. 4b) in comparison to the spectrum for virgin PMHS95 (Fig. 4a). One residual $\mathrm{SiH}$ peak (less than 5\%), corresponding to unreacted $\mathrm{SiH}$ groups, is found shifted towards lower frequencies $\left(2161 \mathrm{~cm}^{-1}\right)$ due to the inductive effect of alkylsubstituted silicon neighboring subunits. The appearance of new $v_{\mathrm{CH}}$ stretching bands in the $2850-2960 \mathrm{~cm}^{-1}$ region confirms bonding of 1-dodecene to $\mathrm{Si}-\mathrm{H}$. Moreover, the single intense and sharp $\delta_{\mathrm{SiCH}_{3}}$ band at $1262 \mathrm{~cm}^{-1}$ for PMHS95 is split at 1259-1268 indicating the appearance of a new $\delta_{\mathrm{SiCH}_{2}}$ band (symmetrical $\mathrm{CH}_{2}$ deformation) confirming that the $\mathrm{Si}-\mathrm{CH}_{2}$-alkyl groups are bonded to the polysiloxane backbone. Other weak bands characteristic of the methylene group are detected at 1466 (scissoring $\left.\delta \mathrm{CH}_{2}\right)$ and in the $1410-1300 \mathrm{~cm}^{-1}$ region $\left(\delta \mathrm{SiCH}_{2}, \delta \mathrm{CH}_{2}\right.$ and $\delta \mathrm{CH}_{3}$ alkyl deformation).

To analyze quantitatively the FTIR spectra after the hydrosilylation reaction, the spectrum of the dodecyl-functionalized thin film in Fig. 4b was compared with that corresponding to the unreacted PMHS95 thin film in Fig. 4a. Note that the unreacted sample used for comparison was dried and washed with chloroform before reaction in order to extract the fraction of soluble polymer (see Experimental Part). Moreover, comparison of the FTIR spectra of the virgin PMHS thin films after this curing process shows no difference, indicating that the $\mathrm{SiH}$ content and $\mathrm{D}^{\mathrm{H}} / \mathrm{T}^{\mathrm{H}}$ ratio are preserved in thin films and that eventual formation of new crosslinks (see below) is not observed before the hydrosilylation reaction.

After hydrosilylation, the $v_{\mathrm{SiOSi}}$ asymmetric stretching band is similar to that of the virgin PMHS95 thin film: in the spectrum of the dodecyl-functionalized sample, the lower frequency of the 
doublet pattern is slightly shifted at $1026 \mathrm{~cm}^{-1}$ and is more intense than for virgin PMHS95. This indicates that the density of $\mathrm{Si}-\mathrm{O}$ bonds does not increase significantly after the reaction with 1dodecene. However, other effects such as the molecular orientation of the polysiloxane segment or crystallization of the alkyl side chains could explain that the SiOSi doublet pattern has different broadening and intensity in comparison to virgin PMHS and alkyl functionalized PMHS.

Under the optimum conditions presented here (concentration of 1-alkene, swelling solvent, times, catalyst etc.), we have shown that the intensities of the higher frequency $1100-\mathrm{cm}^{-1} v_{\mathrm{SiOSi}}$ bands in the functionalized and corresponding virgin thin film samples can be exactly superposed (Fig. 4). This was not the case for the control reaction carried out without adding alkene, especially if the solvent used contains traces of water. In that case, hydrolysis of $\mathrm{SiH}$ to $\mathrm{SiOH}$ by $\mathrm{H}_{2} \mathrm{O}$ can occur giving ultimately new $\mathrm{Si}-\mathrm{O}$ bonds and crosslinks in the network by the Pt-catalyzed dehydrocoupling reaction of $\mathrm{SiH} / \mathrm{SiOH}$ or by $\mathrm{SiOH}$ condensation between different molecules (e.g. $\mathrm{SiCH}_{3} \mathrm{O}_{3 / 2}$ by hydrolysis of $\mathrm{SiHCH}_{3} \mathrm{O}_{2 / 2}$ ), hence leading to an increase in SiOSi bands. With 1alkene, these side-reactions are clearly insignificant because the rate of the primary hydrosilylation reaction (1) was higher given the concentration of alkene and catalyst used. This was further confirmed by the solid state NMR spectrum of the corresponding functionalized powdered gel (see below). Because of the absence of free $\mathrm{Si}-\mathrm{OH}$ groups in the fully condensed virgin PMHS95, the formation of new crosslinks by a Pt-catalyzed secondary reaction between $\mathrm{SiH}$ and $\mathrm{SiOH}$ is insignificant.

\section{Solid State NMR spectroscopy}

We next confirmed by solid state NMR spectroscopy that effective quantitative hydrosilylation of $\mathrm{SiH}$ groups occurred within the loosely crosslinked PMHS95 network for the corresponding dodecyl and octadecyl functionalized powders. Indeed, all NMR data (Figures 5 and 6) support the structural model presented in Figure 3 where the siloxane network is almost fully functionalized with alkyl moieties.

The ${ }^{29}$ Si MAS NMR spectrum of the dodecyl functionalized PMHS95 powder (Figure 5) clearly shows one major peak at $-23.1 \mathrm{ppm}$ characteristics of an (alkyl) $\mathrm{SiCH}_{3} \mathrm{O}_{2 / 2}$ environment for $\mathrm{D}_{2}$ subunits, ${ }^{35}$ demonstrating that dodecene reacted with the $\mathrm{Si}-\mathrm{H}$ bond of the PMHS segment. 
Similarly, the presence of a $\mathrm{T}_{3}$ signal at $-67.8 \mathrm{ppm}$ characteristic of the (alkyl) $\mathrm{SiO}_{3 / 2}$ environment indicates that some crosslinker subunits are functionalized with alkyl chains. Note that the $\mathrm{T}_{3}$ signal includes also $\mathrm{CH}_{3} \mathrm{SiO}_{3 / 2}$ subunits as new crosslink points due to the partial cleavage of $\mathrm{Si}-\mathrm{H}$ bonds of the DH precursor.

In contrast to crosslinked thin films, the formation of new crosslinks is clearly shown by NMR for the powdered gels. This phenomenon can occur resulting from the presence of moisture during the reaction of the alkoxide precursors and drying of the gel under ambient conditions as previously shown for powders. ${ }^{10}$ However, new crosslink formation is probably limited during the preparation of films by spin-coating because gelification and solvent drying is done quickly under nitrogen (see Experimental Part). The content of $\mathrm{T}_{3}$ in the virgin PMHS95 powdered gel was measured independently from the ${ }^{29} \mathrm{Si}$ NMR MAS spectra (not shown) showing a minor $\mathrm{T}_{3}$ peak representing about $7 \%$ of the total silicon NMR signal. Thus, the $\mathrm{T}_{3}$ signal of the dodecyl functionalized powder (about $10 \%$ of the total) results from both methyl and dodecyl substituted $\mathrm{T}_{3}$ silicon species due, respectively, to hydrolysis of DH subunits and dodecyl hydrosilylation of TH crosslinkers.

The residual unreacted $\mathrm{HSiCH}_{3} \mathrm{O}_{2 / 2}$ in $\mathrm{PMHS}$ segments and $\mathrm{HSiO}_{3 / 2}$ in crosslinker subunits are found, respectively, at $-38.2 \mathrm{ppm}\left(\mathrm{D}_{2}{ }^{\mathrm{H}}: 3 \%\right)$ and $-86.0 \mathrm{ppm}\left(\mathrm{T}_{3}{ }^{\mathrm{H}}: 2 \%\right)$ with a characteristic strong ${ }^{1} \mathrm{~J}\left({ }^{29} \mathrm{Si}-\mathrm{H}\right)$ coupling ${ }^{35}$ of $\sim 300 \mathrm{~Hz}$. Their total integration represents less than $5 \%$ of the total of silicon indicating that not all reactive $\mathrm{Si}-\mathrm{H}$ bonds participate in the reaction. The relatively high proportion of unreacted $\mathrm{T}_{3}{ }^{\mathrm{H}}$ subunit $(2 \%)$ indicates that addition of alkene to $\mathrm{SiH}$ bonds of the $\mathrm{TH}$ crosslinker is less favorable. The minor peak $(2 \%)$ at $-57.6 \mathrm{ppm}\left(\mathrm{T}_{2}{ }^{\mathrm{OH}}\right)$ is attributed to the $\mathrm{HOSiCH}_{3} \mathrm{O}_{2 / 2}$ subunit derived from partial hydrolysis of the $\mathrm{DH}$ precursor. This side-reaction can occur during platinum catalytic hydrosilylation with traces of water present in the reaction mixture but is not significant. Similar conclusions are obtained from the ${ }^{29} \mathrm{Si}$ NMR MAS spectra of the octadecyl functionalized PMHS95 powder (not shown) except that as the length of the alkyl chains increases, peak broadening is observed.

The ${ }^{1}$ H MAS NMR spectrum of the dodecyl functionalized PMHS95 powder exhibits the relevant signals of the dodecyl side chain protons at $\delta-0.31 \mathrm{ppm}(\mathrm{Ha}), 0.16(\mathrm{Hb}), 0.50(\mathrm{Hc})$ and $0.90(\mathrm{Hd})$ 
(Figure 6). In particular, the resonance $(\mathrm{Hb})$ at $\delta 0.16 \mathrm{ppm}$ confirms that the $\mathrm{Si}-\mathrm{CH}_{2}$-alkyl groups were successfully bonded to the PMHS polysiloxane backbone. Integration of the ${ }^{1} \mathrm{H}$ NMR signals (b), (c) and (d) using the Gauss/Lorentz fitting procedure is consistent with the presence of $\mathrm{CH}_{3}\left(\mathrm{CH}_{2}\right)_{11} \mathrm{Si}$ moieties. No other signal was detected in the $\mathrm{Si}-\mathrm{CH}$ region $(1.1-1.3 \mathrm{ppm})^{40}$ corresponding to Markovnikov addition of 1-alkene to the $\mathrm{Si}-\mathrm{H}$ bond. If present, the signal must overlap with other signals. As expected for a long alkyl chain, the reaction goes predominantly according to the anti-Markovnikov rule. No $\mathrm{SiH}$ resonance in the $4.2-4.6 \mathrm{ppm}$ region ${ }^{35}$ due to $\mathrm{SiH}$ in DH or TH subunits, was detected confirming that full addition had occurred.

\section{Structural Characterization of the Alkyl Chain Organization}

The arrangement of the alkyl chains in the PMHS network was examined by IR spectroscopy. The existence of crystalline domains was also confirmed by wide angle X-ray diffraction and by DSC analysis.

Table 3 presents the assignment of the characteristic $\mathrm{C}-\mathrm{H}$ stretching modes for the symmetric and antisymmetric stretching vibrations of the $\mathrm{CH}_{2}$ and $\mathrm{CH}_{3}$ groups in the high-frequency region of $n$ alkyl functionalized PMHS95 powders and thin-films. For the octadecyl powder, the positions of the $\mathrm{CH}_{2}$ stretching mode peaks (2851 and $2920 \mathrm{~cm}^{-1}$ ), indicate the presence of an ordered arrangement for the alkyl chains. ${ }^{33,41,42}$ In comparison, the values increase significantly for the dodecyl powder (2854 and $2924 \mathrm{~cm}^{-1}$ ) and for hexyl thin films (2859 and $2926 \mathrm{~cm}^{-1}$ ) indicating a liquid-like behaviour in these materials. In the case of the PMHS70 substrate, $v_{a}\left(\mathrm{CH}_{2}\right)$ is shifted to higher frequencies in functionalized thin films [hexyl (2928), dodecyl (2927), octadecyl (2926)] and in the corresponding powders [hexyl (2926), octadecyl (2928)], showing that the chains are disordered whatever their length. In the highly crosslinked PMHS70 network, the SiOSi framework which is more rigid than in PMHS95 does not favor alkyl chain packing even in the case of long octadecyl chains. The functionalization of the lightly crosslinked PMHS network is thus a pre-requisite for driving the organization of the long alkyl chains in this type of material.

Figure 7 shows the assignments of the observed frequencies to the vibrational modes in the lowfrequency region for octadecyl and dodecyl PMHS95 powders. The appearance of the $\mathrm{CH}_{2}$ rocking 
mode at $720 \mathrm{~cm}^{-1}$ and the $\mathrm{CH}_{2}$ scissoring deformation mode at $1467.5 \mathrm{~cm}^{-1}$ both as sharp peaks with full width at half-maximum (fwhm) values of $\approx 8$ and $12 \mathrm{~cm}^{-1}$, respectively, for the octadecyl network (Fig. 7a), are both indicative of crystalline chain packing with triclinic or hexagonal structures. $^{41,43,44}$

In addition, the observation of a series of progression bands in the $\mathrm{CH}_{2}$ wagging region $\left(1170-1380 \mathrm{~cm}^{-1}\right)$ in the spectrum of the octadecyl powder (Fig. 7a) is consistent with the above analysis indicating the presence of conformationally ordered alkyl chains ${ }^{43,44}$ in agreement with the X-ray diffraction results (see below). In comparison, the spectrum of the dodecyl PMHS95 network shows a broad envelope in these spectral regions (Fig. 7b) suggesting that most of the alkyl chains are in a disordered state.

Similar conclusions are obtained from the spectra of the octadecyl (not shown) and dodecyl (Fig. 4b) surface-grafted PMHS95 thin films of except that we believe that the side chains are less well organized in the thin films than in the unconstrained 3D gels because the polysiloxane backbone becomes more rigid in the constrained stress environment induced by surface attachment.

The packing mode of the alkyl side chains shows a strong resemblance to the similarly constructed materials resulting from amphiphilic segregation such as the products reported using polymerized $n$ octadecyltrichlorosilane in polyoctadecylsiloxane (POS) polymer $^{43,45}$ or in self assembled monolayers (SAM). ${ }^{45,46}$

X-ray diffraction also gives some evidence of the crystalline organization of the $n$-alkyl chains in the PMHS95 network. The X-ray diffraction curves of octadecyl and dodecyl functionalized powders (Figure 8) show diffraction peaks at low angles as well as one peak at large angles. For the octadecyl functionalized powder (Fig. 8a) three harmonics are observed at d-spacings of 29.35, 14.65, and $9.81 \AA$, respectively. It is generally recognized that the observed series of d-spacing in the low-angle region is characteristic of alkyl side chain packing affording layered structures. However, we are not yet able to answer the question as to whether the side chains are interdigitated or if have an end-to-end arrangement. We assume that only the alkyl chains are involved in the formation of crystalline domains since the polysiloxane backbone is known to be a highly flexible polymer. 
The peaks at low angles can thus be related - but not in a one-to-one fashion - to the length of the chains. For the simplest model involving a layered structure in which the alkyl chains are not interdigitated and are perpendicular to the polysiloxane backbone, the largest d-spacing was calculated as twice the molecular length of the fully extended $\mathrm{C}_{18} \mathrm{H}_{37} \mathrm{SiO}$ subunits estimated to be about $2 \times 26 \AA=52 \AA$. The present experimental result was $29 \AA$, being significantly shorter than the calculated value (52 $\AA$ ). This suggests that the $n$-octadecyl chains are partially interdigitated or organized in an inclined arrangement. The first peak of the diffraction pattern of the dodecyl functionalized powder appears at a somewhat higher angle than that for the octadecyl (Fig. 8b), hence at a lower d-spacing, $22 \AA$, in accordance with the shorter length of the molecular chain thus validating the above hypothesis. The second and third harmonics are in this case not visible because of the much broader peaks for the dodecyl powder.

Another possibility is that the three observed peaks are indexed as (002), (004), and (006), respectively, because of the presence of certain crystallographic symmetry elements such as a screw axis causing the systematic extinction of the odd $(00 l)$ reflections. In this case the cell parameter is not $29.35 \AA$ but rather $58.70 \AA$ for the octadecyl functionalized powder, which is closer to twice the estimated fully extended molecular chain length $(52 \AA)$.

The large angle peak is related to the interchain spacing: for dodecyl this peak is at $2 \Theta=19.55^{\circ}$ ( $d$ $=4.53 \AA)$ and for octadecyl it is at $2 \Theta=21.30^{\circ}(\mathrm{d}=4.17 \AA)$ and the fwhm of the peaks are $5.24^{\circ}$ and $1.63^{\circ}$ for dodecyl and octadecyl, respectively. The value of $4.17 \AA$ is within the range observed for dense packing of octadecyl chains such as in the X-ray pattern for POS structures $(4.14 \AA$ ) reported by Parikh et al. ${ }^{43}$ In dodecyl functionalized PMHS95, a broad peak is observed with a higher interband distance of $4.53 \AA$ indicating that amorphous domains in which the dodecyl chains are in the molten state can coexist with crystalline domains. The ability of the side chains to crystallize increases as expected with the alkyl chain length.

Figure 9 shows the DSC curves of the dodecyl C12 and octadecyl C18 powders with side chains of 12 or 18 carbon atoms, respectively. The peak maximum temperatures and enthalpy $\Delta H$ from the DSC thermograms (Fig. 9) are listed in Table 4. Both curves reveal an endothermic transition in the heating process for which the temperature and $\Delta H$ values increase with the length of the side chain. 
The phase transition is attributed to the melting of the side chains in the polymeric network. For the octadecyl powder, the melting peak temperature for the $\mathrm{C} 18$ side chain is $50{ }^{\circ} \mathrm{C}$ higher than that obtained for the C12 side chain in the PMHS95 network because the ability of the side chains to crystallize in the network increases with alkyl chain length, as reported for comb-like polymers. ${ }^{47}$ An evaluation of the percentage of crystallinity $\left(X_{c}\right)$ was attempted by dividing $\Delta H$ with the number of carbons in the aliphatic side chain and by the enthalpy value for the crystallized methylene groups $\Delta H_{c}=3.07 \mathrm{~kJ} / \mathrm{mol}$ per $\mathrm{CH}_{2}$ unit, estimated for the alkane (Table 4). As expected, these networked polymers are composed of amorphous and crystalline domains with higher crystallinity for the longer alkyl chains. In contrast to the functionalized PMHS95 networks, the comb-like poly(n-alkyl methylsiloxane)s with various alkyl side groups obtained by hydrosilylation do not crystallize even in the case of the octadecyl chain. ${ }^{40}$ This indicates that the side chains can crystallize more easily if they are confined in the interior of the crosslinked PMHS95 network in which the mesh size or the PMHS segment have a maximum average length of $43 \AA$ (Table 1).

The melting peak of the $\mathrm{C} 18$ side group $\left(38{ }^{\circ} \mathrm{C}\right.$ in the second heating run) is broad and the shoulder peak reveals a double-melting behavior indicating a different organization mode of the alkyl chains. The first heating curve (not shown) shows multiple endothermic peaks in the temperature range $35-50{ }^{\circ} \mathrm{C}$. The highest peak was found at $47^{\circ} \mathrm{C}$ and it disappeared in the second DSC run. This phenomenon indicates that alkyl chain crystallization in the as-prepared powder will depend on its thermal history. The temperature of the melting transition of the C18 powder is above room temperature confirming the observation of crystalline reflections by X-ray diffraction. For the $\mathrm{C} 12$ powder, minor melting peaks were also observed in the same temperature range $35-50{ }^{\circ} \mathrm{C}$ in the first run (not shown) which had not completely disappeared in the second heating run, as shown by the arrow in Figure 9b. The melting temperature of these minor peaks is quite similar to the observed melting peaks in the $\mathrm{C} 18$ powder. Thus, we attribute these melting peaks to the formation of various $\mathrm{C} 12$ side chain crystals with a more compact structure than the one observed at lower temperature. This confirms the coexistence of amorphous and crystalline phases for the $\mathrm{C} 12$ powders as observed at room temperature by X-ray diffraction. Further studies should be undertaken in order 
to characterize the crystallization of the alkyl side chain as a function of temperature in such networked materials.

\section{CONCLUSIONS}

Polymethylhydrosiloxane materials as thin film or as powdered gels were prepared via sol-gel copolycondensation of $\mathrm{DH} / \mathrm{TH}$ mixtures of silicon hydride alkoxide precursors to provide homogeneous crosslinked PMHS networks for subsequent hydrosilylation of the $\mathrm{SiH}$ groups within the polymer network. FTIR spectroscopy indicates that the $\mathrm{SiH}$ functions are preserved in the crosslinked PMHS thin films and that the crosslink density is controlled by decreasing the concentration of the $\mathrm{TH}$ crosslinker to as low as $1 \%$ (mol \%). The crosslink density determined the mesh size of the gel and the stability of the surface-grafted network. The soft elastomeric organic films with crosslinking ratio (TH mol \%) less than 5 to $1 \%$ were indeed surface-attached and cannot be removed from the surface even by rigorous extraction with boiling solvent $\left(\mathrm{CHCl}_{3}\right.$, toluene, hexane). Hence, subsequent hydrosilylation of the PMHS95 samples ( $c$. $1 \mu \mathrm{m})$ with olefins gives $n$ alkyl functionalized thin films without delamination. The reaction of the $\mathrm{SiH}$ sites in such elastomeric networks was almost quantitative for olefin as the number of carbons in the alkyl chain was varied between 6 and 18. In contrast to PMHS95, the SiH conversion decreased significantly in rigid PMHS70 on increasing the alkyl chain length from 6 to 18 carbons. This result demonstrates that the facility of functionalization of $\mathrm{SiH}$ sites in the network depends on the crosslink density because the diffusion of alkene molecules is limited. The solid state NMR spectrum of the $n$-dodecyl and n-octadecyl functionalized PMHS95 powders confirmed the network reactivity toward hydrosilylation by the high value for $\mathrm{SiH}$ conversion - more than $95 \%$ of the total amount of silicon - leading to the expected anti-Markovnikov adduct for the long alkyl chains. Also, structural information about the packing arrangement of the side chain is consistent with crystallization of the long alkyl chain in the polymeric network. The DSC melting temperature of $n$-octadecyl and $n$ dodecyl PMHS95 powders were $38^{\circ} \mathrm{C}$ and $-10{ }^{\circ} \mathrm{C}$, respectively. As expected, the ability of the side chains to crystallize increases with alkyl chain length from dodecyl to octadecyl. We believe that sol-gel tailored elastomeric PMHS polymer networks of tunable crosslink density provide an 
interesting and versatile route to surface modification since by taking advantage of the $\mathrm{Si}-\mathrm{H}$ function various organic moieties can be introduced by the hydrosilylation reaction.

\section{Acknowledgements}

M. Barboiu is thanked for discussions and P. Huguet for his advice concerning the infrared spectral analysis. P. Déjardin is thanked for his comments about the manuscript. N. Masquelez is thanked for the thermal analyses, D. Cot for the microscope observations, and E. Petit for his technical assistance. We acknowledge support from the CNRS.

\section{References}

1. Luzinov, I.; Minko, S.; Tsukruk, V. V. Prog Polym Sci 2004, 29, 635-698.

2. Murata, H.; Chang, B. J.; Prucker, O.; Dahm, M.; Ruhe, J. Surf Sci 2004, 570, 111-118.

3. Pluedemann, E. P. Silane Coupling Agents; Plenum Press: New York, 1982.

4. Ulbricht, M. Polymer 2006, 47, 2217-2262.

5. Gualandris, V.; Babonneau, F.; Janicke, M. T.; Chmelka, B. F. J Sol-Gel Sci Technol 1998, 12, $75-80$.

6. Pauthe, M.; Phalippou, J.; Corriu, R.; Leclercq, D.; Vioux, A. J Non-Cryst Solids 1989, 113, 21 30.

7. Schaudel, B.; Guermeur, C.; Sanchez, C.; Nakatani, K.; Delaire, J. A. J Mater Chem 1997, 7, 61-65.

8. Sorarù, G. D.; D'Andrea, G.; Campostrini, R.; Babonneau, F. J Mater Chem 1995, 5, 13631374.

9. Loy, D. A.; Baugher, B. M.; Baugher, C. R.; Schneider, D. A.; Rahimian, K. Chem Mater 2000, $12,3624-3632$.

10. Thami, T.; Bresson, B.; Fretigny, C. J Appl Polym Sci 2007, 104, 1504-1516.

11. Kolel-Veetil, M. K.; Keller, T. M. J Polym Sci Part A: Polym Chem 2006, 44, 147-155.

12. Grunlan, M. A.; Lee, N. S.; Mansfeld, F.; Kus, E.; Finlay, J. A.; Callow, J. A.; Callow, M. E.; Weber, W. P. J Polym Sci Part A: Polym Chem 2006, 44, 2551-2566.

13. Gadda, T. M.; Kus, E.; Mansfeld, F.; Finlay, J. A.; Callow, J. A.; Callow, M. E.; Kowalke, G. L.; Wendt, D. E.; Weber, W. P. J Polym Sci Part A: Polym Chem 2006, 44, 2237-2247. 
14. Srividhya, A.; Reddy, B. S. R. J Polym Sci Part A: Polym Chem 2007, 45, 1707-1726.

15. Brzoska, J. B.; Azouz, I. B.; Rondelez, F. Langmuir 1994, 10, 4367-4373.

16. Evmenenko, G.; Yu, C. J.; Kmetko, J.; Dutta, P. Langmuir 2002, 18, 5468-5472.

17. Cosgrove, T.; Patel, A.; Semlyen, J. A.; Webster, J. R. P.; Zarbakhsh, A. Langmuir 1993, 9, 2326-2329.

18. Tada, H.; Nakamura, K.; Nagayama, H. J Phys Chem 1994, 98, 12452-12457.

19. Helmy, R.; Wenslow, R. W.; Fadeev, A. Y. J Am Chem Soc 2004, 126, 7595-7600.

20. Lukevics, E.; Belyakova, Z. V.; Pomerantseva, M. G.; Voronkov, M. G. J Organomet Chem Libr 1977, 5, 1-179.

21. Speier, J. L. Adv Organomet Chem 1979, 17, 407-447.

22. Stein, J.; Lewis, L. N.; Gao, Y.; Scott, R. A. J Am Chem Soc 1999, 121, 3693-3703.

23. Pesek, J. J.; Matyska, M. T. Interface Sci 1997, 5, 103-117.

24. Sandoval, J. E.; Pesek, J. J. Anal Chem 1991, 63, 2634-2641.

25. Sandoval, J. E.; Pesek, J. J. Anal Chem 1989, 61, 2067-2075.

26. Pesek, J. J.; Matyska, M. T.; Prabhakaran, S. J. J Separ Sci 2005, 28, 2437-2443.

27. Ketelson, H. A.; Brook, M. A.; Pelton, R. H. Chem Mater 1995, 7, 1376-1383.

28. Ketelson, H. A.; Brook, M. A.; Pelton, R.; Heng, Y. M. Chem Mater 1996, 8, 2195-\&.

29. Ketelson, H. A.; Pelton, R.; Brook, M. A. Colloids and Surfaces A: Physicochem Eng Aspects 1998, 132, 229-239.

30. Kobayashi, S.; Tanaka, I.; Shirota, O.; Kanda, T.; Ohtsu, Y. J Chromatogr A 1998, 828, 75-81.

31. Suhara, T.; Kanemaru, T.; Fukui, H.; Yamaguchi, M. Colloids and Surfaces A: Physicochem Eng Aspects 1995, 95, 1-9.

32. Suhara, T.; Kutsuna, H.; Fukui, H.; Yamaguchi, M. Colloid Polym Sci 1993, 271, 486-493.

33. Ulman, A. Ultrathin Organic Films; Academic Press: New York, 1991.

34. Edward, P. Handbook of Optical Constants of Solids; Academic Press: London, 1985.

35. Smith, A. L. Chemical Analysis, Vol. 112: The Analytical Chemistry of Silicones; Wiley: New York, 1991. 
36. Lin-Vien, D.; Colthup, N. B.; Fateley, W. G.; Grasselli, J. G. The Handbook of Infrared and Raman Characteristic Frequencies of Organic Molecules; Academic Press: Boston, 1991.

37. Frye, C. L.; Collins, W. T. J Am Chem Soc 1970, 92, 5586-5588.

38. Legrand, A. P. The surface properties of silicas; Wiley: Chichester, 1998.

39. Toomey, R.; Freidank, D.; Ruhe, J. Macromolecules 2004, 37, 882-887.

40. Mukbaniani, O.; Titvinidze, G.; Tatrishvili, T.; Mukbaniani, N.; Brostow, W.; Pietkiewicz, D. J Appl Polym Sci 2007, 104, 1176-1183.

41. Macphail, R. A.; Strauss, H. L.; Snyder, R. G.; Elliger, C. A. J Phys Chem 1984, 88, 334-341.

42. Snyder, R. G.; Strauss, H. L.; Elliger, C. A. J Phys Chem 1982, 86, 5145-5150.

43. Parikh, A. N.; Schivley, M. A.; Koo, E.; Seshadri, K.; Aurentz, D.; Mueller, K.; Allara, D. L. J Am Chem Soc 1997, 119, 3135-3143.

44. Snyder, R. G. J Chem Phys 1967, 47, 1316-1360.

45. Wang, R. W.; Baran, G.; Wunder, S. L. Langmuir 2000, 16, 6298-6305.

46. Allara, D. L.; Parikh, A. N.; Rondelez, F. Langmuir 1995, 11, 2357-2360.

47. Jordan, E. F.; Feldeise D. W.; Wrigley, A. N. J Polym Sci Part A1: Polym Chem 1971, 9, 1835-1851.

\section{FIGURE LEGENDS}

Figure 1. Transmission infrared spectra of crosslinked PMHS thin films (ca. $1 \mu \mathrm{m})$ as a function of the DH/TH composition: 50/50, 70/30, and 90/10 (mol \%).

Figure 2. $\mathrm{Si}-\mathrm{H}$ stretching peak position $v_{\mathrm{SiH}}$ of $\mathrm{SiHCH}_{3} \mathrm{O}_{2 / 2}$ subunit as a function of the $\mathrm{DH}$ molar fraction in crosslinked PMHS thin films $(c a .1 \mu \mathrm{m})$. The straight line corresponds to the least square fit of the experimental data: $v_{\max }\left(\mathrm{cm}^{-1}\right)=-24.7 \alpha+2192.7$ where $\alpha$ is the DH molar fraction.

Figure 3. Schematic representation of the lightly crosslinked PMHS network structure and complete olefin addition to the $\mathrm{SiH}$ active sites via catalytic hydrosilylation. $N$ is the average number of repeat units per PMHS segment between trifunctional $\mathrm{SiHO}_{3 / 2}$ crosslinks. The swollen structure of the network after olefin functionalization is depicted by a volume change normal to the surface. 
Figure 4. Transmission FTIR spectra of (a) virgin PMHS95 thin film $(1.1 \mu \mathrm{m})$, and (b) after dodecyl functionalization via catalytic hydrosilylation (same sample).

Figure 5. ${ }^{29} \mathrm{Si}-\mathrm{NMR}$ MAS spectrum of dodecyl functionalized PMHS95 powder. Single pulse experiment was done with proton decoupling, 500 scans accumulation.

Figure 6. ${ }^{1} \mathrm{H}-\mathrm{NMR}$ MAS spectrum of dodecyl functionalized PMHS95 powder, single pulse experiment, 32 scans accumulation.

Figure 7. Transmission FTIR spectra of $n$-alkyl functionalized PMHS95 powder in $\mathrm{KBr}$ matrix for (a) n-octadecyl powder and (b) n-dodecyl powder.

Figure 8. X-ray patterns for powder samples of $n$-alkyl functionalized PMHS95 network for (a) $n$ octadecyl powder, and (b) $n$-dodecyl powder. Interlayer d-spacing $(00 l)$ is given in nanometers.

Figure 9. DSC second heating scans of $n$-alkyl functionalized PMHS95 network for (a) $n$-octadecyl powder and (b) $n$-dodecyl powder, run at $10{ }^{\circ} \mathrm{C} /$ min under $\mathrm{N}_{2}$.

\section{GRAPHICAL ABSTRACT:}

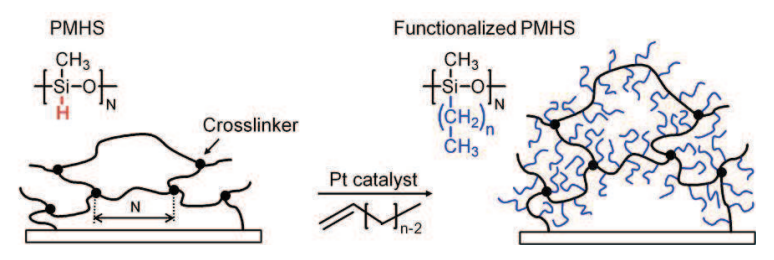

Surface attached thin films of a polymethylhydrosiloxane (PMHS) network were deposited by a room temperature sol-gel process and were thereafter functionalized by hydrosilylation of the $\mathrm{SiH}$ groups with 1-alkenes $\mathrm{CH}_{2}=\mathrm{CH}\left(\mathrm{CH}_{2}\right)_{\mathrm{n}-2} \mathrm{CH}_{3}$. Postfunctionalization of PMHS gives a polysiloxane network with $n$-alkyl side chains. The influence of structural factors on the extent of $\mathrm{SiH}$ functionalization, such as the size of the alkenes and the mesh size of the network, was studied in relation to the crosslinking ratio. We obtained effective quantitative conversion of $\mathrm{SiH}$ within the 5\% crosslinked PMHS network. The functionalized networks show crystallization of the long $n$-alkyl side chains. 


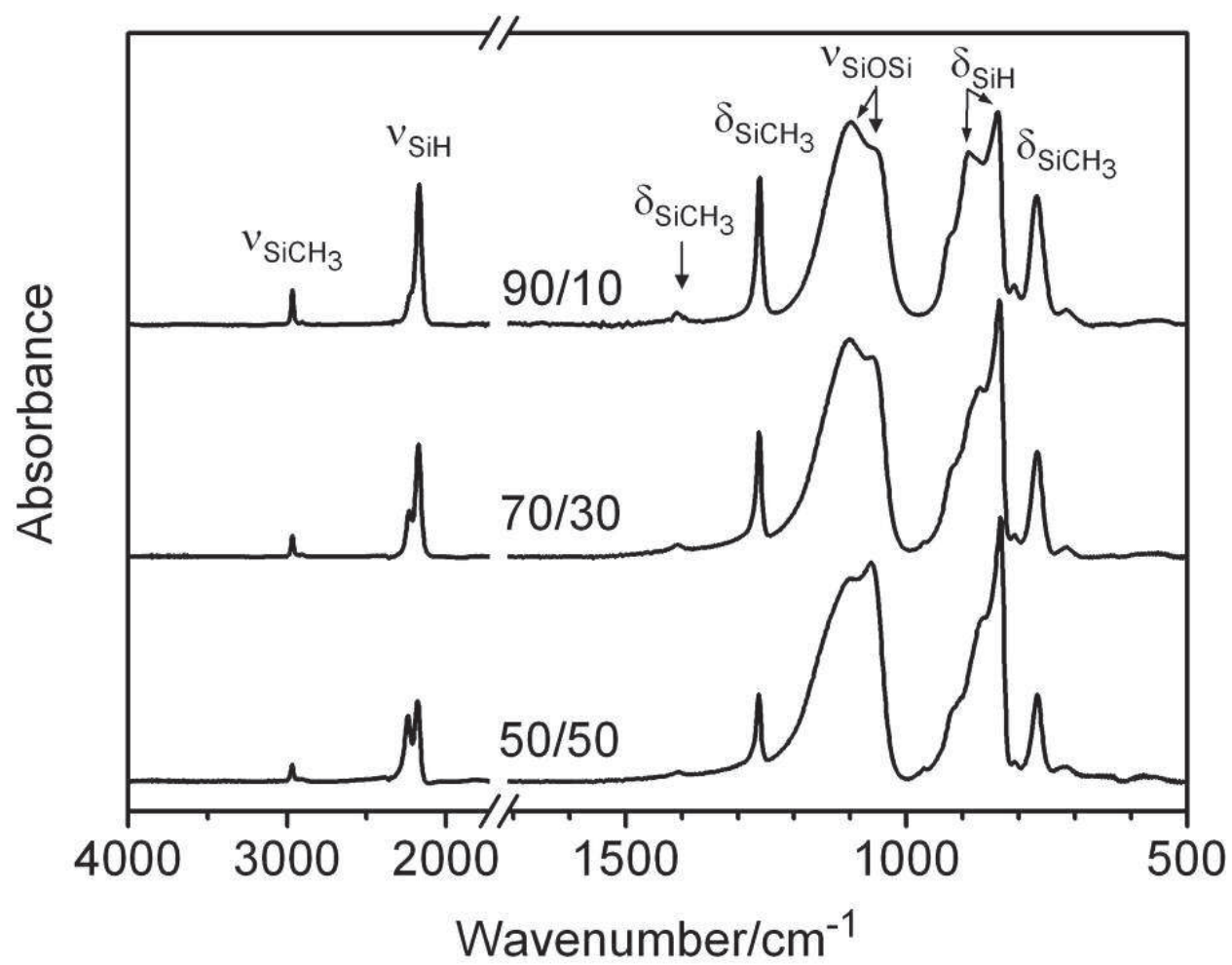

Figure 1. Transmission infrared spectra of crosslinked PMHS thin films (ca. $1 \mu \mathrm{m}$ ) as a function of the DH/TH composition: 50/50, 70/30, and 90/10 ( $\mathrm{mol} \%$ ). $80 \times 63 \mathrm{~mm}(300 \times 300 \mathrm{DPI})$ 


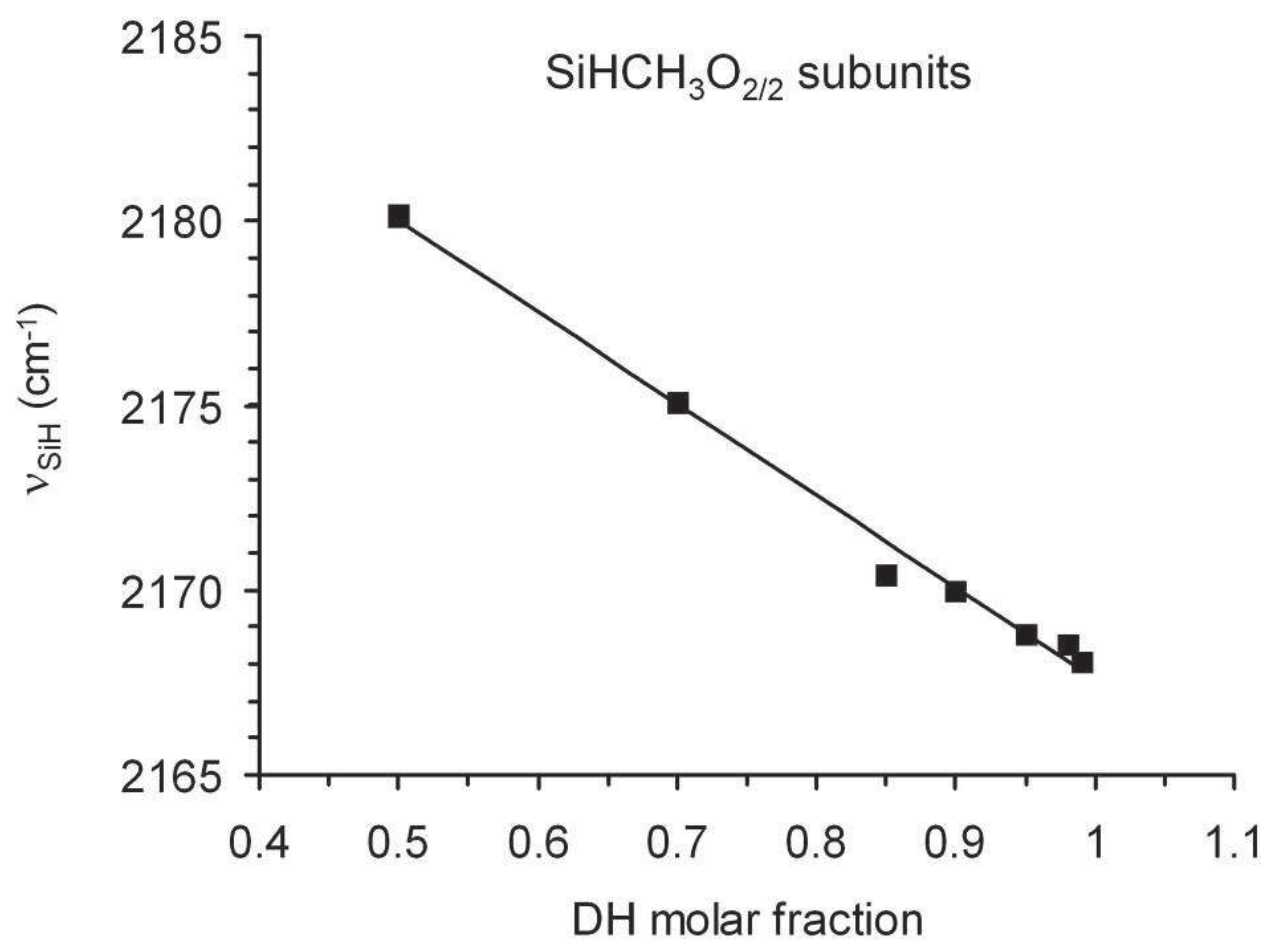

Figure 2. Si-H stretching peak position $\mathrm{V}$ SiH of $\mathrm{SiHCH}_{3} \mathrm{O}_{2 / 2}$ subunit as a function of the DH molar fraction in crosslinked PMHS thin films (ca. $1 \mu_{\mathrm{m}}$ ). The straight line corresponds to the least square fit of the experimental data: $v_{\max }\left(\mathrm{cm}^{-1}\right)=-24.7 \alpha+$ 2192.7 where $\alpha_{\text {is }}$ the DH molar fraction. $80 \times 60 \mathrm{~mm}(300 \times 300 \mathrm{DPI})$ 
PMHS<smiles>C[Si](C)(C)O[Si](C)(C)C</smiles>

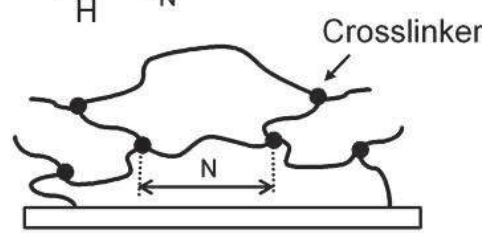

Functionalized PMHS

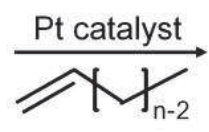

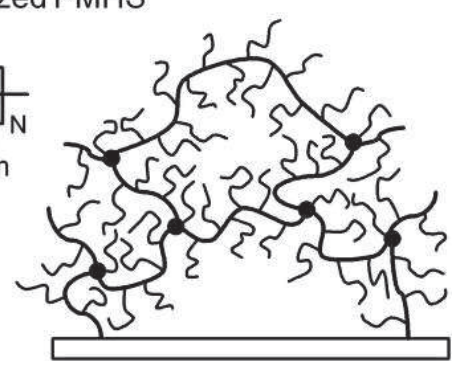

Figure 3. Schematic representation of lightly crosslinked PMHS network structure and complete olefin addition to the $\mathbf{S i H}$ active sites via catalytic hydrosilylation. $\mathbf{N}$ is the average number of repeat units per PMHS segment between trifunctional $\mathrm{SiHO}_{3 / 2}$ crosslinks. The swollen structure of the network after olefin functionalization is depicted by a volume change normal to the surface. $80 \times 25 \mathrm{~mm}(300 \times 300 \mathrm{DPI})$ 

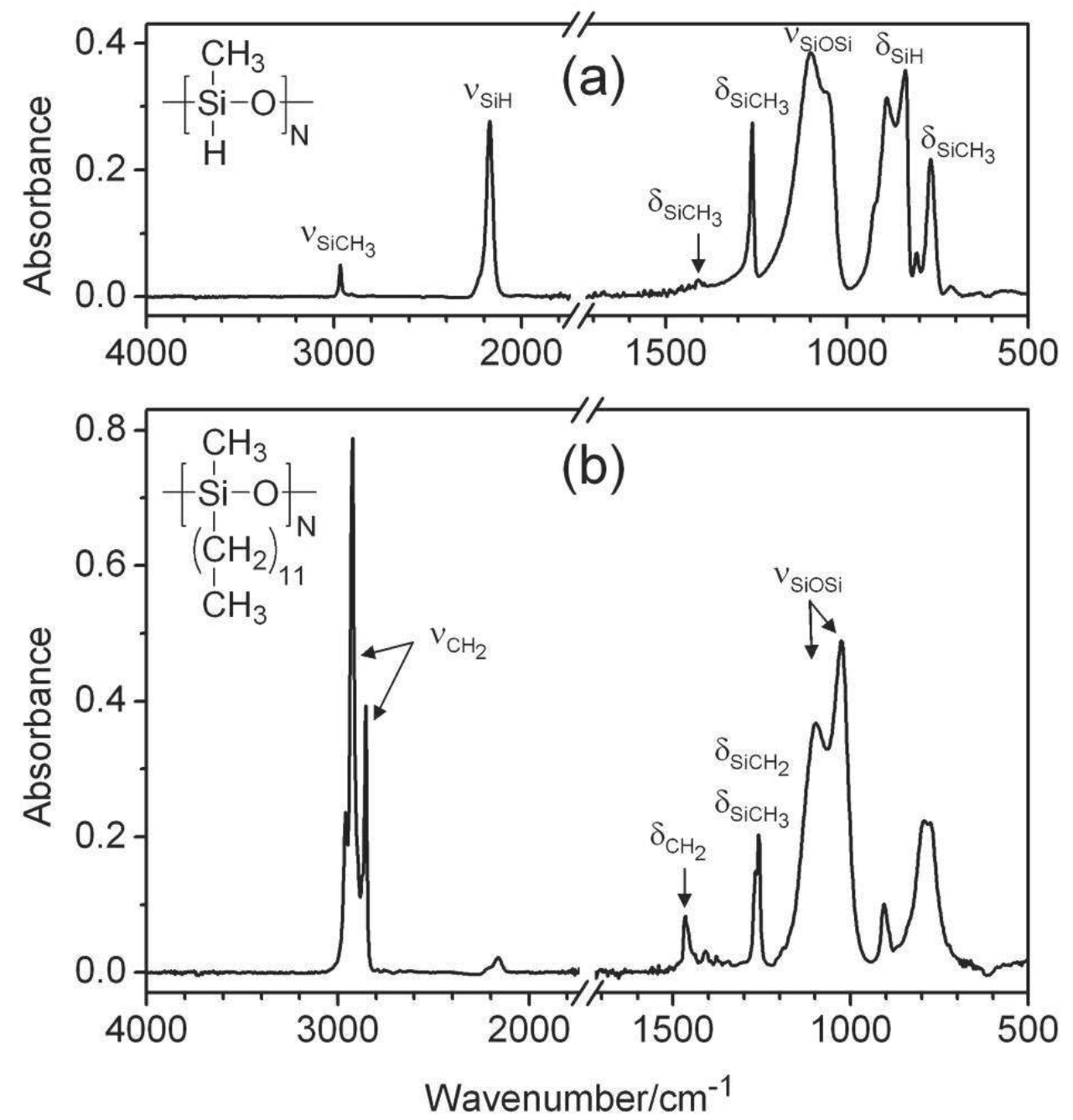

Figure 4. Transmission FTIR spectra of (a) virgin PMHS95 thin film $\left(1.1 \mu_{\mathrm{m}}\right.$ ) and (b) after dodecyl functionalization via catalytic hydrosilylation (same sample). $80 \times 85 \mathrm{~mm}(300 \times 300$ DPI $)$ 


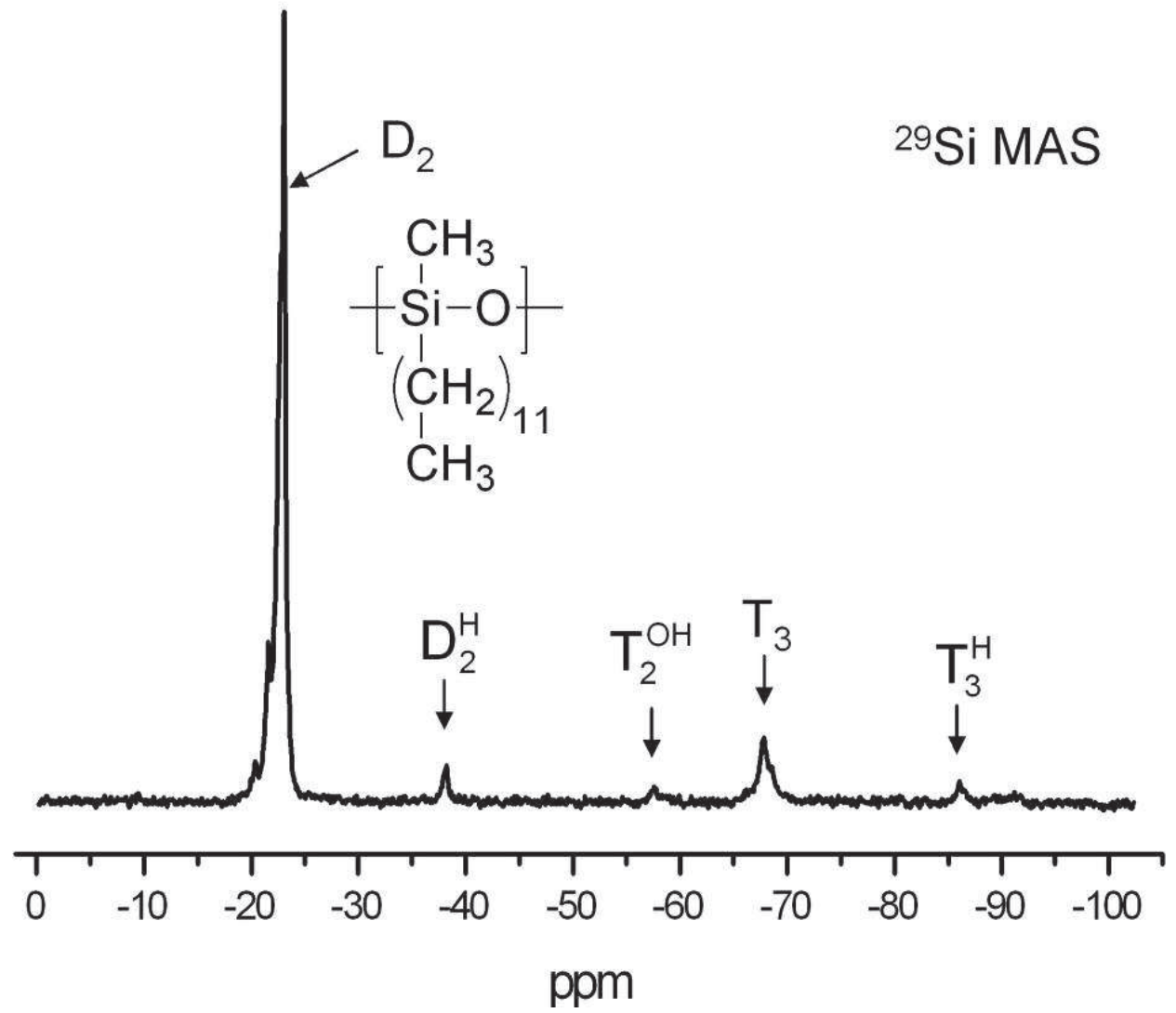

Figure 5. ${ }^{29}$ Si-NMR MAS spectrum of dodecyl functionalized PMHS95 powder. Single pulse experiment was done with proton decoupling, 500 scans accumulation. $80 \times 69 \mathrm{~mm}(300 \times 300 \mathrm{DPI})$ 


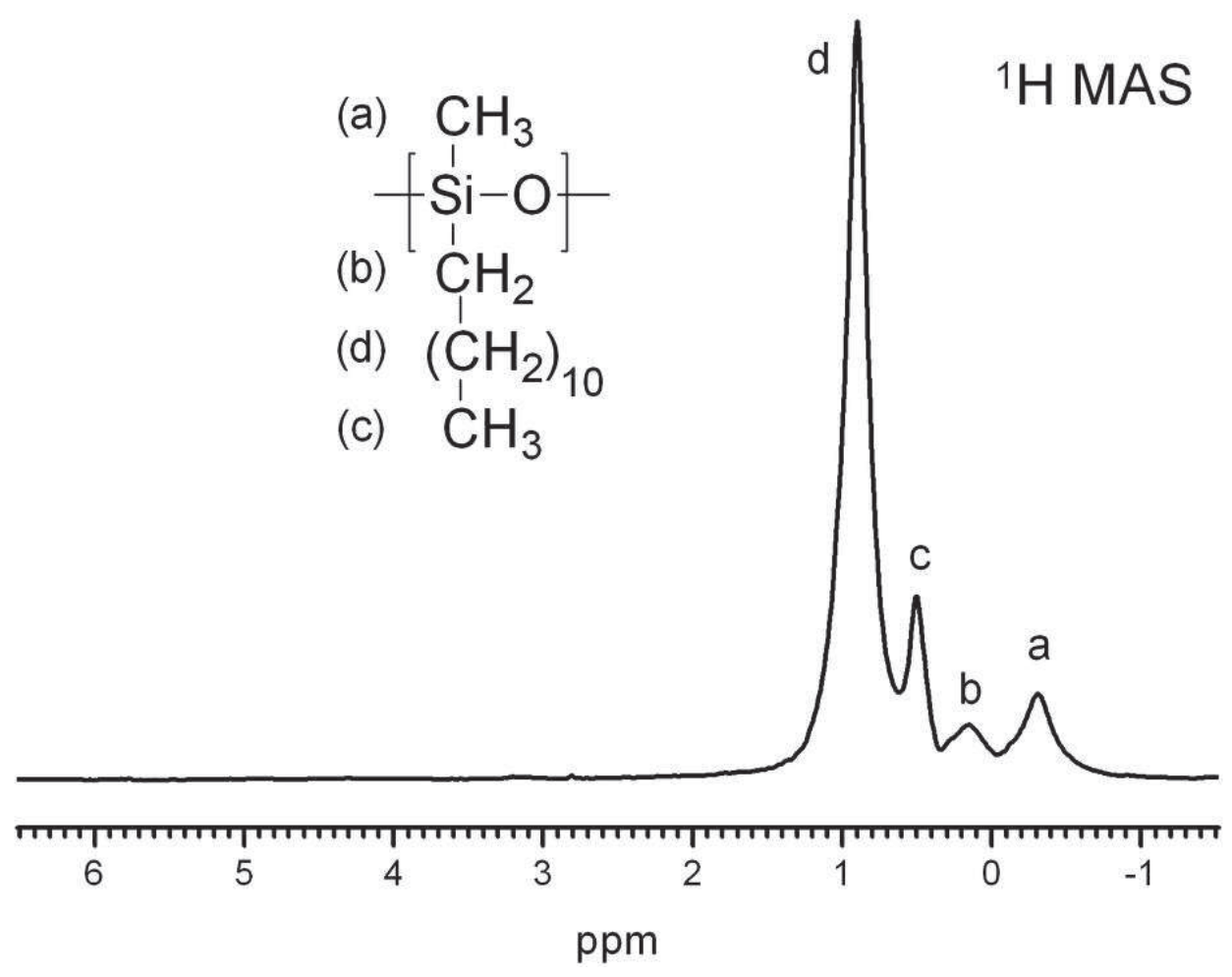

Figure 6. ${ }^{1}$ H-NMR MAS spectrum of dodecyl functionalized PMHS95 powder, single pulse experiment, 32 scans accumulation. $80 \times 62 \mathrm{~mm}(300 \times 300$ DPI) 


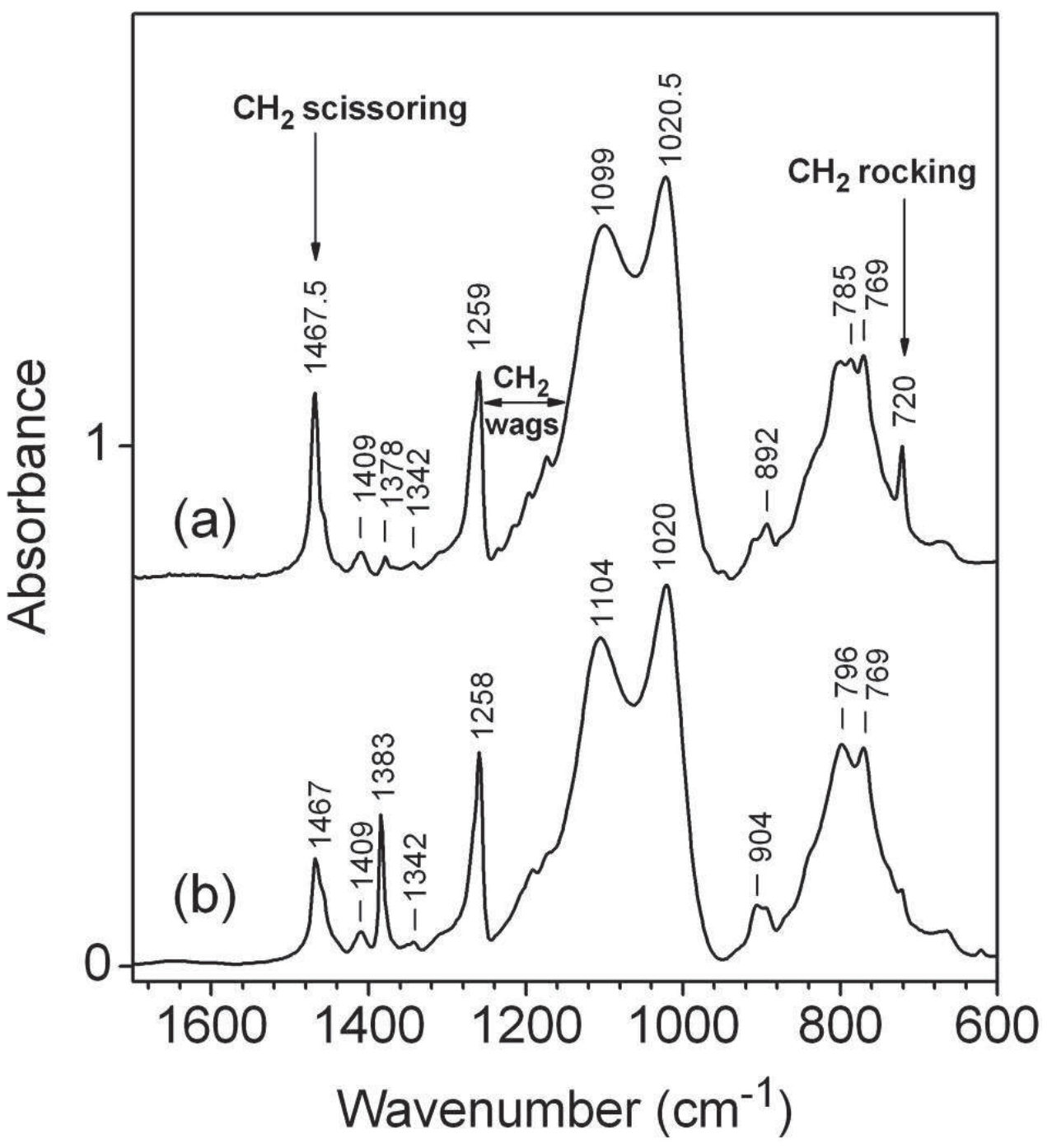

Figure 7. Transmission FTIR spectra of alkyl functionalized PMHS95 powder in KBr matrix: (a) n-octadecyl powder; (b) n-dodecyl powder. $80 \times 88 \mathrm{~mm}(300 \times 300$ DPI $)$ 


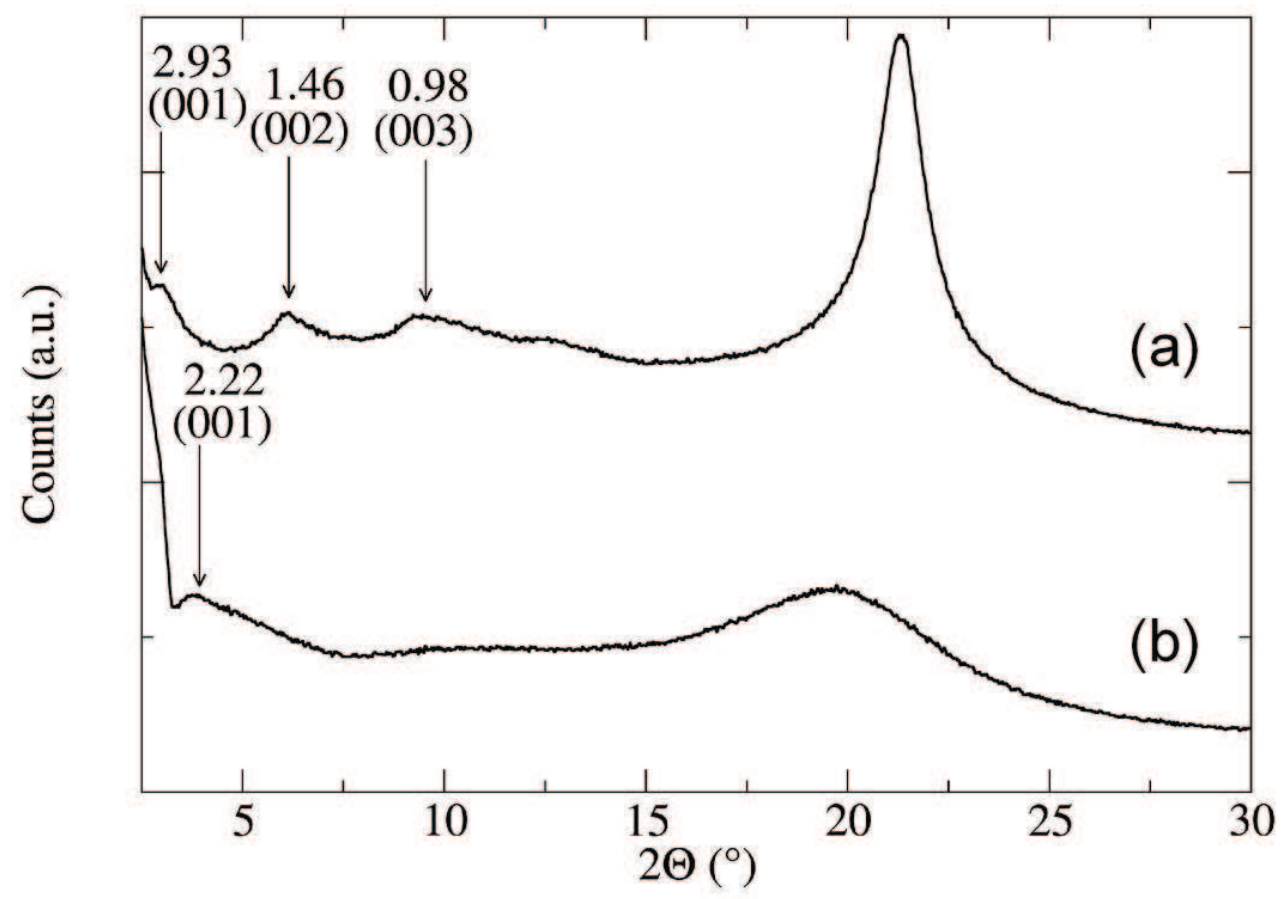

Figure 8. X-ray patterns for powder samples of $n$-alkyl functionalized PMHS95 network: (a) $n$-octadecyl powder; (b) $n$-dodecyl powder. Interlayer d-spacing $(00 /)$ is given in nanometers.

$89 \times 63 \mathrm{~mm}(300 \times 300$ DPI $)$ 


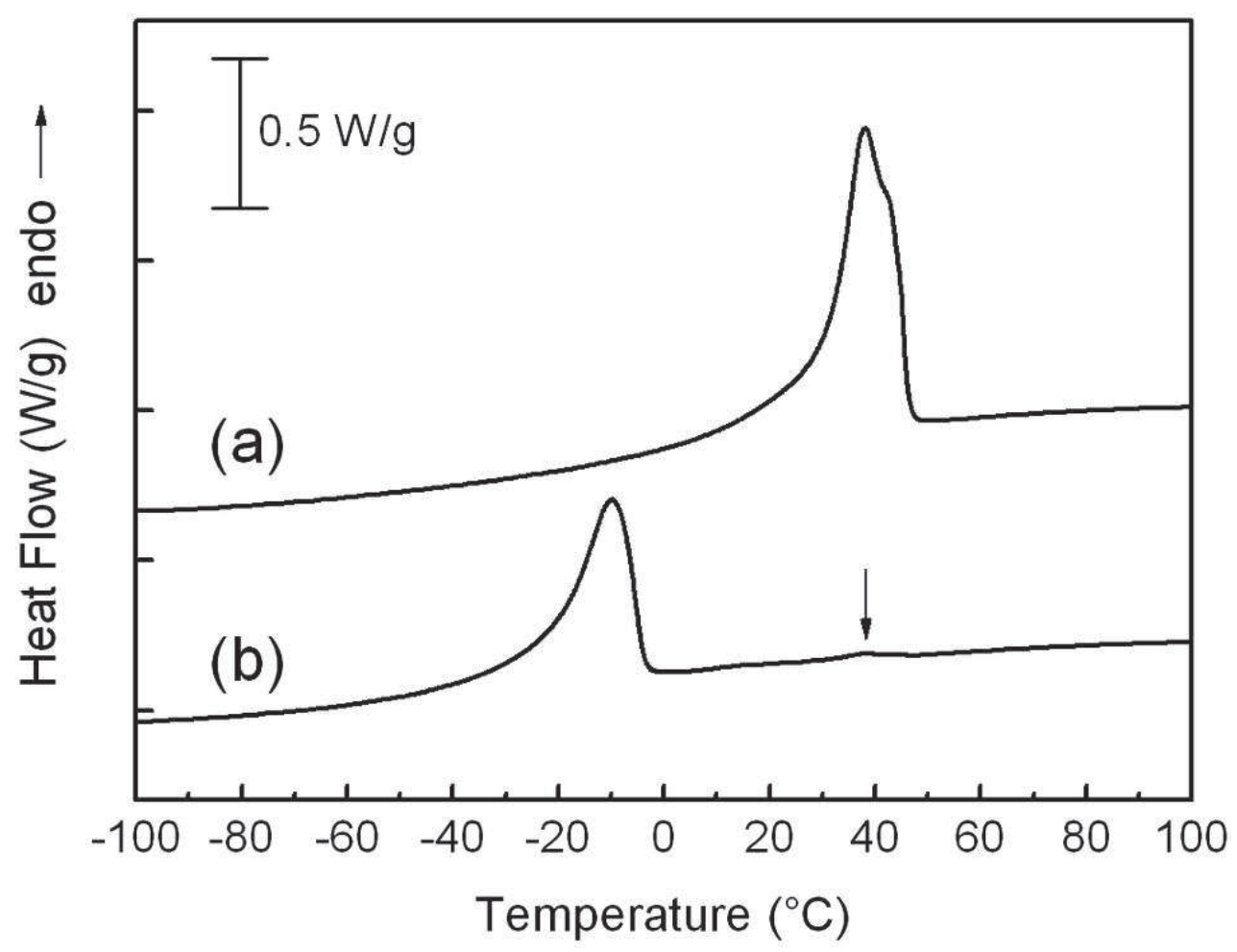

Figure 9. DSC second heating scans of n-alkyl functionalized PMHS95 network for (a) noctadecyl powder and (b) n-dodecyl powder, run at $10^{\circ} \mathrm{C} / \mathrm{min}$ under $\mathbf{N}_{2}$. $80 \times 62 \mathrm{~mm}(300 \times 300$ DPI $)$ 
Table 1. Typical properties of silicon wafer grafted DH/TH thin films with most characteristic FTIR data (Accuracy $\pm 0.1 \mathrm{~cm}^{-1}$ ) and calculated values of the average number of repeat subunits DH between crosslinks in PMHS segments $(N)$ and of the upper limit mesh size of the PMHS network $\left(\xi_{\max }\right)$.

\begin{tabular}{lllllll}
\hline Composition & Thickness $^{\mathrm{a}}$ & TH subunit & DH subunit & DH subunit & $N$ & $\begin{array}{c}\xi_{\max } \\
\text { DH/TH }(\mathrm{mol} \%)\end{array}$ \\
\hline & $(\mu \mathrm{m})$ & $v_{\mathrm{SiH}}\left(\mathrm{cm}^{-1}\right)$ & $v_{\mathrm{SiH}}\left(\mathrm{cm}^{-1}\right)$ & $\delta_{\mathrm{SiCH}_{3}}\left(\mathrm{~cm}^{-1}\right)$ & & $(\AA)$ \\
\hline $50 / 50$ & $0.15-1.0$ & 2242.7 & 2180.1 & 1263.5 & 0.7 & 5.2 \\
$70 / 30$ & $0.01-1.0$ & 2233.7 & 2175.1 & 1262.8 & 1.6 & 8.0 \\
$85 / 5$ & $1.1-1.3$ & $2222 \mathrm{sh}$ & 2170.4 & 1262.1 & 3.8 & 15 \\
$90 / 10$ & $1.0-1.1$ & $\mathrm{sh}$ & 2169.6 & 1261.9 & 6.0 & 22 \\
$95 / 5$ & $1.1-1.2$ & $\mathrm{sh}$ & 2168.8 & 1261.8 & 13 & 43 \\
$98 / 2$ & $0.7-0.8$ & $\mathrm{sh}$ & 2168.5 & 1261.7 & 33 & 105 \\
$99 / 1$ & $0.8-1.0$ & $\mathrm{sh}$ & 2168.1 & 1261.7 & 66 & 209 \\
\hline
\end{tabular}

a on the basis of FTIR calibration and ellipsometry measurements. Sol-gel conditions were $\mathrm{EtOH}($ molar ratio $[\mathrm{EtOH}] /[\mathrm{Si}]=1)$; hydrolysis ratio $h=\left[\mathrm{H}_{2} \mathrm{O}\right] /[\mathrm{SiOEt}]=0.5$; catalyst content $\left[\mathrm{CF}_{3} \mathrm{SO}_{3} \mathrm{H}\right] /[\mathrm{Si}]=0.05 \mathrm{~mol} / \mathrm{mol}(\%)$; using dip-coating or spin-coating methods.

sh $=$ shoulder band 
Table 2. Spectroscopic measurement of $\mathrm{SiH}$ conversion (\%) for olefin hydrosilylation of PMHS network using the Karstedt catalyst (except as mentioned) and effect of the anchored alkyl group $\mathrm{Si}-\left(\mathrm{CH}_{2}\right)_{\mathrm{n}} \mathrm{CH}_{3}$ on thin films and on powdered gels.

\section{$\mathrm{SiH}$ conversion $(\%)$}

Anchored group $\quad$ Hexyl $(n=5) \quad$ Dodecyl $(n=11) \quad$ Octadecyl $(n=17)$

\section{PMHS95 substrate}

Film $(100 \mathrm{~nm} \text { to } 1 \mu \mathrm{m})^{(\mathrm{a})}$

Powder ${ }^{(c)}$

PMHS70 substrate

Film $(150 \mathrm{~nm})^{(\mathrm{a})}$ 95

Film $(10 \mathrm{~nm})^{(\mathrm{d})}$ 70

Powder $^{(\mathrm{d})}$$$
-
$$

65

$$
\sim 100
$$

$95^{(b)}$

95

90

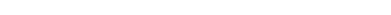

75

20

60

$-$

${ }^{\text {a }} \mathrm{SiH}$ conversion (\%) measured on the basis of FTIR absorption measurements at the $\mathrm{Si}-\mathrm{H}$ stretching band frequency by measuring total integrated intensities in the $2100-2300 \mathrm{~cm}^{-1}$ region of unreacted and reacted thin film samples.

${ }^{\mathrm{b}}$ Using the Speier catalyst yielded same results.

${ }^{\mathrm{c}}$ From solid state ${ }^{29} \mathrm{Si}$ NMR: Residual $\mathrm{SiH}$ is less than $5 \%$ of the total of silicon signal.

${ }^{\mathrm{d}} \mathrm{SiH}$ conversion $(\%)$ based on $\mathrm{v}_{\mathrm{a}}\left(\mathrm{CH}_{2}\right)$ intensities. 
Table 3. Vibrational assignments and peak positions $\left(\mathrm{cm}^{-1}\right)$ of $\mathrm{C}-\mathrm{H}$ stretching symmetric $v_{\mathrm{s}}$ and antisymmetric $v_{\mathrm{a}}$ vibrations in transmission infrared spectra of $n$-alkyl functionalized PMHS95 thin film on wafer and corresponding powder as a function of the $n$-alkyl side chain $\mathrm{Si}-\left(\mathrm{CH}_{2}\right)_{\mathrm{n}} \mathrm{CH}_{3}$.

\begin{tabular}{llllll}
\hline & \multicolumn{3}{c}{ PMHS95 Thin film } & \multicolumn{2}{c}{ PMHS95 Powder } \\
Anchored group & $\begin{array}{l}\text { Hexyl } \\
(n=5)\end{array}$ & $\begin{array}{l}\text { Dodecyl } \\
(n=11)\end{array}$ & $\begin{array}{l}\text { Octadecyl } \\
(n=17)\end{array}$ & $\begin{array}{l}\text { Dodecyl } \\
(n=11)\end{array}$ & $\begin{array}{l}\text { Octadecyl } \\
(n=17)\end{array}$ \\
\hline$v_{\mathrm{a}}\left(\mathrm{CH}_{2}\right)$ & 2926 & 2924 & 2921 & 2924 & 2920 \\
$v_{\mathrm{s}}\left(\mathrm{CH}_{2}\right)$ & 2859 & 2854 & 2851 & 2854 & 2851 \\
$v_{\mathrm{a}}\left(\mathrm{CH}_{3}\right)$ & 2960 & 2958 & 2957 & 2959 & 2957 \\
$v_{\mathrm{s}}\left(\mathrm{CH}_{3}\right)$ & 2874 & sh & 2872 & sh & sh \\
\hline
\end{tabular}

sh $=$ shoulder band 
Table 4. Side chains melting temperature, enthalpy $\Delta H$ and crystallinity $X_{c}$ obtained from the DSC endothermic peak of $n$-octadecyl and $n$-dodecyl functionalized PMHS95 powders.

\begin{tabular}{llll}
\hline & Second run & & \\
Anchored group & DSC peak $\left({ }^{\circ} \mathrm{C}\right)$ & $\Delta H(\mathrm{~kJ} / \mathrm{mol})$ & $X_{c}(\%)$ \\
\hline Octadecyl $(n=17)$ & 38.0 & -26.0 & 47 \\
Dodecyl $(n=11)$ & -10.1 & -11.2 & 30 \\
\hline
\end{tabular}




\title{
SUPPORTING INFORMATION
}

\section{Functionalization of Surface-Grafted Polymethylhydrosiloxane Thin Films with Alkyl Side Chains}

\author{
Thierry Thami ${ }^{1}$, Gihane Nasr ${ }^{1}$, Hanan Bestal ${ }^{1}$, Arie van der Lee ${ }^{1}$ and Bruno Bresson ${ }^{2}$ \\ ${ }^{1}$ Institut Européen des Membranes, ENSCM, Université Montpellier 2, CNRS, CC047, 2 Place \\ Eugène Bataillon, 34095 Montpellier Cedex 5 \\ ${ }^{2}$ LPQ/ESPCI, CNRS, 10 rue Vauquelin, 75231 Paris cedex 05
}

\section{RESULTS AND DISCUSSION}

\section{Synthesis and Characterization of Crosslinked PMHS Thin Films}

\section{Quantitative Analysis of the $\mathrm{v}_{\mathrm{SiH}}$ Band by Infrared Spectroscopy}

To analyze quantitatively the FTIR spectra as a function of the DH molar composition, the $\delta_{\mathrm{SiCH}_{3}}$ $\left(1262 \mathrm{~cm}^{-1}\right)$ and $v_{\mathrm{SiH}}\left(2275 \mathrm{~cm}^{-1}\right)$ peaks of the DH subunit were chosen because they are sharp and intense for DH molar fraction above $50 \%$. These peaks were integrated to determine the peak area given by $\int A(v) d v$ over the region of interest where $A$, and $v$ are the absorbance and the wavenumber of the peak. For the doublet-like $\mathrm{SiH}$ structure, a Gauss-Lorentz fitting procedure was used to obtain the area of the $\mathrm{DH}$ peak. The areas of the $\mathrm{SiH}$ and $\mathrm{SiCH}_{3}$ peaks of the $\mathrm{DH}$ subunit were normalized by using the total integrated intensity of the $\mathrm{SiH}$ bands of the $\mathrm{DH}$ and $\mathrm{TH}$ subunits in the $2100-2300 \mathrm{~cm}^{-1}$ region where no other band-overlapping can occur (Figure S1).

A series of thin films of comparable thicknesses of about $1 \mu \mathrm{m}$ was chosen for the quantitation with DH molar fraction ranging from 50\% to $95 \%$. Figure S1 shows that the normalized peak area of the methyl $\delta_{\mathrm{SiCH}_{3}}$ and of $v_{\mathrm{SiH}}$ stretching bands of $\mathrm{SiHCH}_{3} \mathrm{O}_{2 / 2}$ subunits versus the $\mathrm{DH}$ molar fraction 
are straight lines passing through the origin. The above analysis indicates quantitatively that the $\mathrm{SiH}$ groups are mostly preserved in the $1 \mu \mathrm{m}$-thick film.

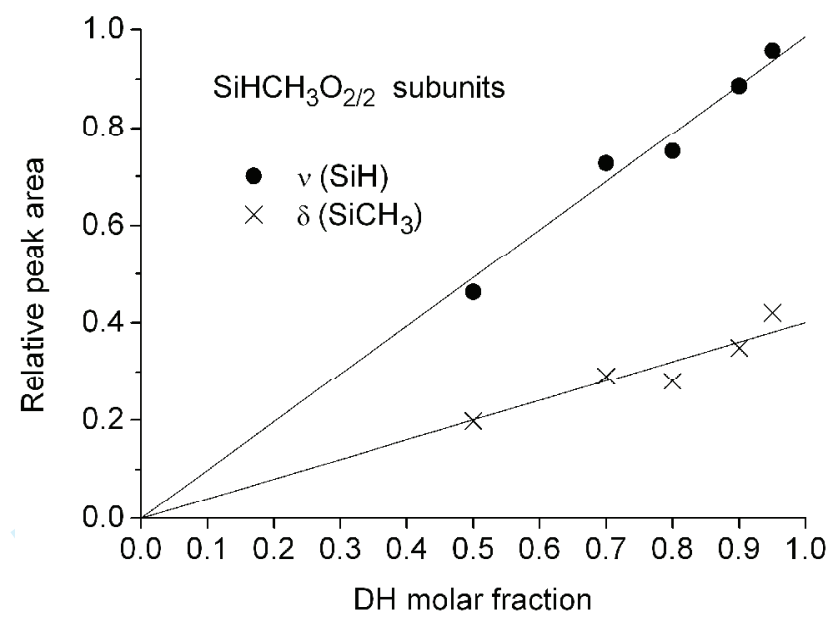

Figure S1. Relative peak area of the symmetrical deformation $\delta_{\mathrm{SiCH}_{3}}\left(1262 \mathrm{~cm}^{-1}\right)$ and of the stretching $v_{\mathrm{SiH}}\left(2275 \mathrm{~cm}^{-1}\right)$ of $\mathrm{SiHCH}_{3} \mathrm{O}_{2 / 2}$ subunits versus the molar fraction of the $\mathrm{DH}$ precursor in thin films. We normalized the peak area using the total integrated intensity of the $2100-2300 \mathrm{~cm}^{-1}$ $\mathrm{SiH}$ bands whose value of about $10 \mathrm{~cm}^{-1}$ corresponds to thin films of comparable thicknesses (ca. 1 $\mu \mathrm{m})$. The straight line corresponds to least square fit.

\section{Gauss-Lorentz, Fitting Procedure of $v_{S i H}$ Band Structure by Infrared Spectroscopy}

Analysis of the $\mathrm{SiH}$ stretching peaks in the $2100-2300 \mathrm{~cm}^{-1}$ region was performed using the Gauss-Lorentz fitting procedure (LabSpec Software); the quality of the fitting was evaluated on the basis of the $\chi^{2}$ values (about $10^{-7}$ ) and correlation coefficient values $=0.999$.

For instance, a Gauss-Lorentz peak fitting procedure was applied to the FTIR spectra of the PMHS90 thin film (Figure S2). An excellent agreement between the experimental and fitted spectra was achieved with four Gauss/Lorentz curves. The integrated absorbances of the DH curves $(\mathbf{1}+\mathbf{2})$ $89 \%$ and $\mathrm{TH}$ curves $(\mathbf{3}+\mathbf{4}) 11 \%$, normalized to the total area of the $\mathrm{Si}-\mathrm{H}$ band, are in good agreement with the DH/TH 90/10 composition of the sol. 
On the basis of the fitting results, the different frequencies of the shifted curves were attributed to various possible subunits arrangements in the PMHS90 network taking into account the releasing effect of the methyl group (Figure S3). As sketched, the sol-gel preparation scheme should give a broad distribution of $\mathrm{D}^{\mathrm{H}}$ and $\mathrm{T}^{\mathrm{H}}$ subunits with PMHS chains segments having an average number of $\mathrm{D}^{\mathrm{H}}$ repeat units of about 6 . The narrow low shifted curve $1\left(27 \%\right.$ of the total $\mathrm{SiH}$ area) at $2166 \mathrm{~cm}^{-1}$ with a full width at half-maximum (fwhm) value of $25 \mathrm{~cm}^{-1}$, can be attributed to self-condensed DH subunits in the PMHS segment in agreement with values found for homopolymers PMHS $^{1,2}$ while the broad curve $2(62 \%)$ centered at $2174 \mathrm{~cm}^{-1}$ with a fwhm of $44 \mathrm{~cm}^{-1}$ includes various DH subunits co-condensed with TH crosslinker (see Figure S3). This result qualitatively reflects the broad distribution of polysiloxane chains in the PMHS network.

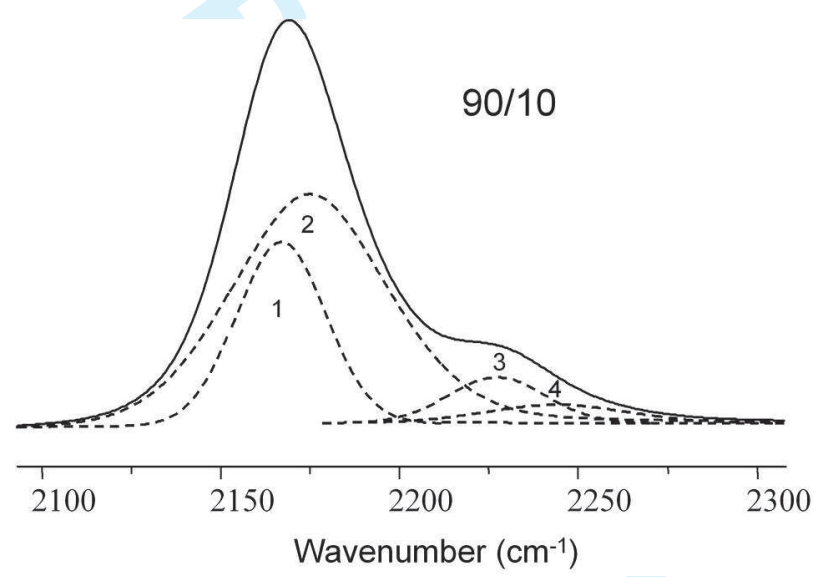

Figure S2. Experimental and Gauss/Lorentz fitted FTIR spectra of the SiH stretching vibration in the 2100-2300 $\mathrm{cm}^{-1}$ region of the PMHS90 thin film obtained from DH/TH 90/10 (mol \%) sol. Typical fitted values of peak positions $v\left(\mathrm{~cm}^{-1}\right)$ and full width at half-maximum (fwhm) values in parenthesis $\left(\mathrm{cm}^{-1}\right)$ are: $\mathbf{1}=2166(25) ; \mathbf{2}=2174(44) ; \mathbf{3}=2227(27) ; \mathbf{4}=2244$ (40). Integrated absorbances of the various curves normalized to $100 \%$ with the total area of $\mathrm{SiH}$ were: $\mathbf{1}(27 \%) ; 2$ $(62 \%) ; 3(7 \%) ; 4(4 \%)$ 


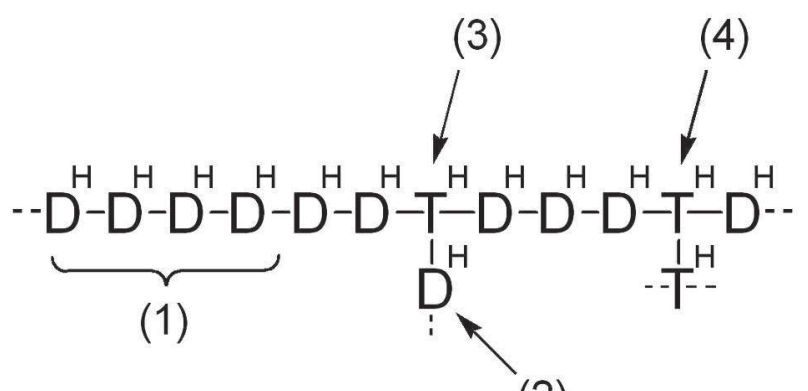

(2)

Figure S3. Schematic view of the various possible microstructural arrangements of the monomeric subunits in a fully $\mathrm{T}^{\mathrm{H}}$-crosslinked PMHS90 network and tentative assignment of the fitted curves (1-4) in Fig. S2. $\mathrm{D}^{\mathrm{H}}$ and $\mathrm{T}^{\mathrm{H}}$ denote respectively the corresponding fully condensed $\mathrm{SiHCH}_{3} \mathrm{O}_{2 / 2}$ and $\mathrm{SiHO}_{3 / 2}$ subunits.

On the other hand, the experimental integrated intensities of curve 1 (30\%) and 2 (70\%) normalized to the total intensity of $\mathrm{DH}$ peak can be compared in good agreement with a statistical model describing a purely random polymerization process where the polymer microstructure can therefore be described by 6 repeat subunits between crosslinks, $\left(\mathrm{T}^{\mathrm{H}}\right)\left(\mathrm{D}^{\mathrm{H}}\right)_{1}\left(\mathrm{D}^{\mathrm{H}}\right)_{2}\left(\mathrm{D}^{\mathrm{H}}\right)_{3}\left(\mathrm{D}^{\mathrm{H}}\right)_{3}\left(\mathrm{D}^{\mathrm{H}}\right)_{2}\left(\mathrm{D}^{\mathrm{H}}\right)_{1}\left(\mathrm{~T}^{\mathrm{H}}\right)$, where the relative proportions of the neighboring $\mathrm{DH}$ subunits of various environments are $\left(\mathrm{D}^{\mathrm{H}}\right)_{1}+\left(\mathrm{D}^{\mathrm{H}}\right)_{2}(67 \%)$ and $\left(\mathrm{D}^{\mathrm{H}}\right)_{3}(33 \%)$.

The $\mathrm{Si}-\mathrm{H}$ band corresponding to the $\mathrm{TH}$ subunits is also fitted with two curves: the low shifted curve $3(7 \%)$ at $2227 \mathrm{~cm}^{-1}$ was attributed to $\mathrm{TH}$ co-condensed with three DH subunits while the curve $4(4 \%)$ centered at $2244 \mathrm{~cm}^{-1}$ corresponds to other co-condensed species. Note that the $\mathrm{SiH}$ peak value for self-condensed $\mathrm{TH}$ gels ${ }^{3}\left(\mathrm{HSiO}_{3 / 2}\right)_{\mathrm{n}}$ prepared under similar conditions or in $\mathrm{T}^{\mathrm{H}}-$ resin $^{4}$ is found at higher frequencies $\left(2260 \mathrm{~cm}^{-1}\right)$. This clearly rules out any strong formation of TH clusters in the PMHS90 gel. Oligomeric species ${ }^{4}$ such as $\mathrm{T}_{8}^{\mathrm{H}}\left(\mathrm{HSiO}_{3 / 2}\right)_{8}\left(2285 \mathrm{~cm}^{-1}\right)$ or $\mathrm{T}_{10}^{\mathrm{H}}\left(\mathrm{HSiO}_{3 / 2}\right)_{10}(2270$ $\mathrm{cm}^{-1}$ ) were not observed in these PMHS90 gels. 


\section{Synthesis and Characterization of Alkyl Functionalized PMHS}

The assignment of the two most intense and characteristic $v_{\mathrm{CH}}$ antisymmetric and symmetric stretching vibration peaks of $\mathrm{CH}_{2}$ groups namely $v_{\mathrm{a}}\left(\mathrm{CH}_{2}\right)$ and $v_{\mathrm{s}}\left(\mathrm{CH}_{2}\right)$ were done at 2921-2926 and 2851-2859 $\mathrm{cm}^{-1}$, respectively, for the various alkyl side chains $\mathrm{Si}-\left(\mathrm{CH}_{2}\right)_{\mathrm{n}} \mathrm{CH}_{3}$ in crosslinked alkyl functionalized PMHS thin films. Their maximum intensity is correlated with the disappearance of $\mathrm{SiH}$ bands. Further, by measuring the ratio of the maximum absorbance of these peaks, relative to the $1100-\mathrm{cm}^{-1} \mathrm{SiOSi}$ band taken as internal reference $\left(A_{\mathrm{CH}_{2}} / A_{\mathrm{SiO}}\right)$, it was found that the ratio follows a quasi linear relationship with the number of methylene group $(n)$ in the hydrocarbon chain $\mathrm{Si}-$ $\left(\mathrm{CH}_{2}\right)_{\mathrm{n}} \mathrm{CH}_{3}$ (Figure $\left.\mathrm{S} 4\right)$.

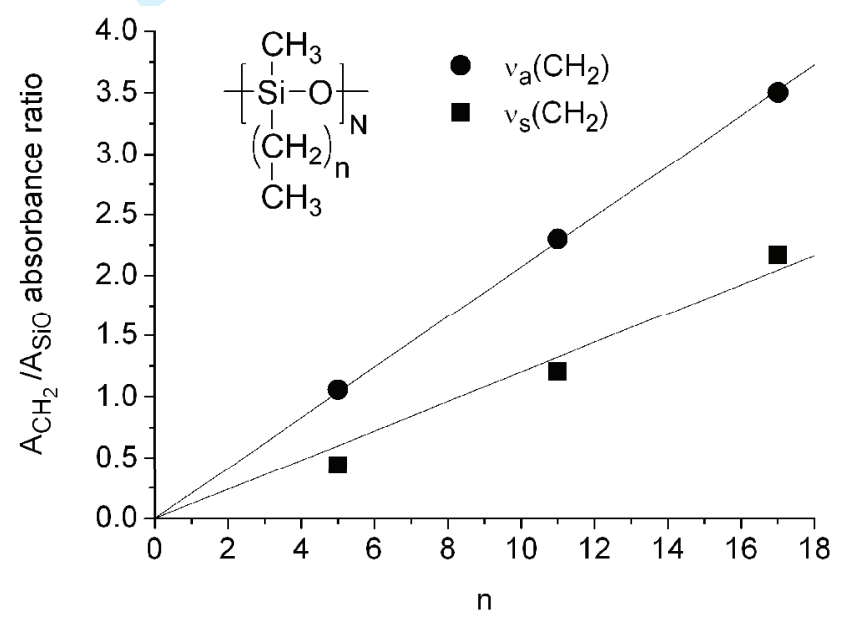

Figure S4. Antisymmetric and symmetric $v_{\mathrm{a}}\left(\mathrm{CH}_{2}\right)$ and $v_{\mathrm{s}}\left(\mathrm{CH}_{2}\right)$ absorbance ratio $A_{\mathrm{CH}_{2}} / A_{\mathrm{SiO}}$ in spectra of alkyl functionalized PMHS95 thin film $(c a .1 \mu \mathrm{m})$ as a function of the number of methylene groups $(n)$ in the alkyl side chain $\mathrm{Si}-\left(\mathrm{CH}_{2}\right)_{\mathrm{n}} \mathrm{CH}_{3} . A_{S i O}$ is the absorbance of the $1100-\mathrm{cm}^{-1} \mathrm{SiOSi}$ band used as internal reference and $A_{C_{2}}$ is the absorbance of the $v_{\mathrm{a}}\left(\mathrm{CH}_{2}\right)$ and $\mathrm{v}_{\mathrm{s}}\left(\mathrm{CH}_{2}\right)$ peaks at 2921-2926 and 2851-2859 $\mathrm{cm}^{-1}$, respectively. For $n=17$, the experimental $\mathrm{CH}_{2}$ intensity was corrected by a factor of 1.1 to take into account that $\mathrm{SiH}$ conversion is $90 \%$.

\section{References}

1. Lin-Vien, D.; Colthup, N. B.; Fateley, W. G.; Grasselli, J. G. The Handbook of Infrared and Raman Characteristic Frequencies of Organic Molecules; Academic Press: Boston, 1991. 
2. Smith, A. L. Chemical Analysis, Vol. 112: The Analytical Chemistry of Silicones; Wiley: New York, 1991.

3. Pauthe, M.; Phalippou, J.; Corriu, R.; Leclercq, D.; Vioux, A. J Non-Cryst Solids 1989, 113, 21 30.

4. Frye, C. L.; Collins, W. T. J Am Chem Soc 1970, 92, 5586-5588. 

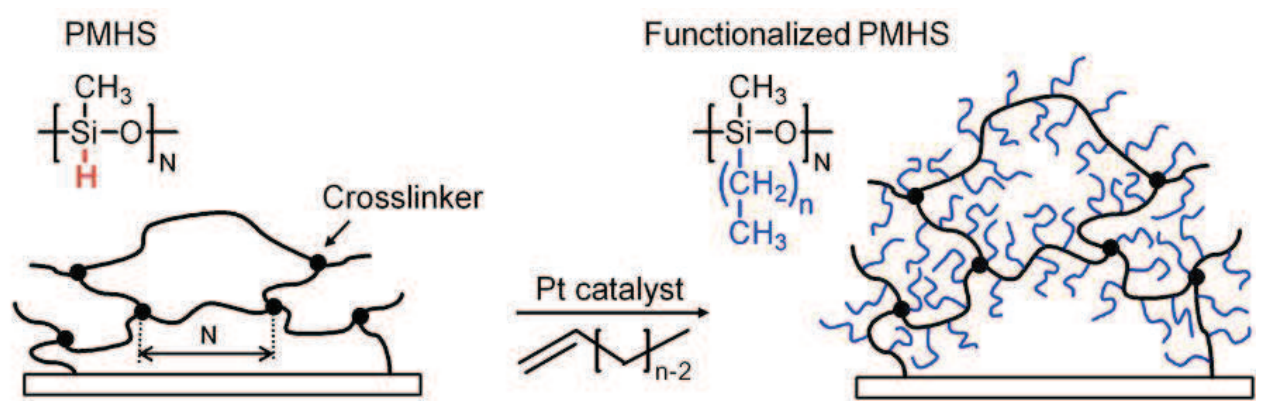

Table of Content

$76 \times 23 \mathrm{~mm}(300 \times 300$ DPI $)$ 